\title{
On consistency and rate of convergence of Flux Reconstruction for time-dependent problems
}

\author{
Kartikey Asthana ${ }^{1}$, Jerry Watkins ${ }^{2}$, Antony Jameson ${ }^{3}$ \\ Department of Aeronautics and Astronautics, Stanford University, CA 94305, USA
}

\begin{abstract}
This study is directed at a rigorous characterization of the consistency and convergence of discontinuous finite element schemes formulated using Flux Reconstruction (FR). We show that the FR formulation is consistent for linear advection and converges to the exact solution for any scheme that is stable in the von Neumann sense. The numerical solution for a scheme of polynomial order $P$ is composed of $P+1$ eignemodes, of which, one and exactly one is 'physical' such that it exhibits the analytical dispersion behavior in the limit of asymptotic grid resolution. The remaining $P$ modes are 'spurious' such that the fraction of energy received by them from the initial condition vanishes in the asymptotic limit. On grid refinement, the rate of convergence of the numerical solution is a function of time, starting from a short-time rate at $t=0^{+}$, associated with interpolation, and asymptotically approaching a long-time rate as $t \rightarrow \infty$, associated with numerical differentiation. Both these rates can be inferred directly from the eigensystem of the numerical derivative operator. We verify these analytical expectations using simple experiments in 1-D and 2-D.
\end{abstract}

Keywords: Flux Reconstruction, Discontinuous Galerkin, consistency, rate of convergence, dispersion, dissipation

2000 MSC: 65M12, 65M15, 65M60

\footnotetext{
${ }^{1} \mathrm{PhD}$, Stanford University. kasthana@alumni.stanford.edu

${ }^{2} \mathrm{PhD}$ candidate, Stanford University. watkins2@stanford.edu

${ }^{3}$ Professor, Department of Aeronautics and Astronautics, Stanford University. jameson@baboon.stanford.edu
}

Preprint submitted to Journal of Computational Physics

December 4, 2016

(C) 2017. This manuscript version is made available under the Elsevier user license

http://www.elsevier.com/open-access/userlicense/1.0/ 


\section{Introduction}

The conventional approach to high-order computing has relied upon compact finite difference schemes $[1,2]$ that are particularly well suited for Cartesian meshes. Although extensions to curvilinear and deforming meshes have been proposed [3], the applicability of such schemes to industrial problems remains limited due to the difficulty involved in generating structured meshes for complex geometries. Consequently, the development of discontinuous finite element methods has been a focal point for recent efforts in the field of computational mechanics. In comparison to traditional finite volume schemes, these methods are capable of attaining higher accuracy through the representation of the solution in an appropriate basis within each element [4]. At the same time, in the case of explicit-time stepping, the provision of discontinuity at the element interfaces allows for an element-local mass matrix which avoids the computational expense associated with continuous finite element schemes [5]. Popular examples within this class of methods include the discontinuous Galerkin (DG) scheme $[6,7,8]$ and the Spectral Difference (SD) scheme [9, 10]. Recently, Huynh [11] proposed a Flux Reconstruction (FR) approach for tensor-product elements that provides a generalized differential framework for recovering both the collocation based nodal DG scheme as well as a version of the SD scheme. This framework has been successfully extended to triangular $[12,13]$ as well as tetrahedral [14] elements. Similar frameworks such as the Correction Procedure via Reconstruction (CPR) [15] have now been proposed that unify the FR and the Lifting Collocation Penalty (LCP) [16] formulations.

The tools for analysis of discontinuous finite element schemes primarily comprise functional analysis and spectral decomposition. In the case of DG, results from functional analysis and approximation theory have been employed to derive a-priori $[17,18,7]$ as well as a-posteriori $[19,20]$ error estimates for linear systems. Superconvergence of DG at the Gauss-Radau points [21] and the $(2 P+1)^{t h}$ order super-accuracy [22], for a $P^{t h}$ degree DG scheme, have been shown. For the FR formulation, use of Lagrangian basis functions has enabled proofs and studies of linear [23] as well as nonlinear [24] stability and led to the definition of energy-stable schemes [25]. On the other hand, Fourier analysis has been primarily utilized to study the dispersive and dissipative properties of numerical schemes. Instances of such studies range from finite difference schemes [1, 26], finite element schemes $[27,28]$, and the DG scheme $[29,30,31,32,33]$. Rigorous studies regard- 
ing the asymptotic behavior of the eigensystem $[34,35]$ have shown that the dispersive and dissipative errors for the DG scheme vanish at a superconvergent rate. The wave properties of the general FR formulation have also been examined $[11,36,37]$ and the effect of scheme parameters such as polynomial order, correction functions, solution points and interface fluxes has been qualitatively explained.

In this paper, we demonstrate that Fourier analysis is sufficient to rigorously develop consistency and convergence theory for discontinuous finite element schemes. In particular, for the linear advection problem, an analytical form of the numerical solution can be prescribed by posing the numerical conservation law as a linear dynamical system. The limit of asymptotic grid resolution can then be inferred from the limiting state of the eigensystem. We show that an a-priori bound on the rate of convergence can be obtained from the convergence rate of the eigenvalues and modal weights of the eigenvectors. These modal weights are associated with the distribution of the initial condition among the eignemodes and consequently depend directly on the location of solution points. Similar to the observations of Cheng and Shu $[38,39]$, who studied the grid-convergence of error for DG, we show that the rate of convergence for any $\mathrm{FR}$ scheme is a function of time, starting from a short-time rate at $t=0^{+}$, associated with interpolation, and asymptotically approaching a long-time rate as $t \rightarrow \infty$, associated with numerical differentiation. Both these rates can be obtained directly from the eigensystem of the numerical derivative operator. Closed-form expressions can be derived for low polynomial orders, $P \leq 3$, as was demonstrated by Guo et al. [40] who utilized symbolic computation for analyzing the DG scheme. However, for $P>3$, we must rely on numerical eigenvalue computations owing to the Abel-Ruffini theorem [41]. Nonetheless, for all polynomial orders of practical relevance, the associated computational effort is negligible.

The paper is formatted as follows. In $\S 2$, we describe the model problem under consideration and the eigensystem associated with the numerical solution. The consistency and convergence of the FR formulation are established in $\S 3$. $\S 4$ then shows that the rate of convergence is a function of time and bounds can be obtained directly from the eigenvalues and modal weights. We end this article by conducting simple numerical experiments in $\S 5$ to verify the analytical expectations. 


\section{Dynamical system for the numerical solution}

This introductory section demonstrates that the numerical solution of the 1-D scalar advection equation using a nodal discontinuous finite element scheme of polynomial order $P$ leads to a linear dynamical system with $P+1$ eigenmodes. The eigenvalues of this system prescribe the numerical dispersion relation and, for upwinded schemes, are associated with characteristic time scales of dissipation. Moreover, expansion of the initial condition in the basis of eigenvectors prescribes the distribution of modal weights among the $P+1$ eigenmodes. Such an eigensystem completely specifies the evolution of error in time.

\subsection{Problem specification}

Consider the linear advection equation in 1-D,

$$
\begin{aligned}
\frac{\partial u}{\partial t}+c \frac{\partial u}{\partial x} & =0, \quad x \in \mathbb{R}, t>0, \\
u(x, 0) & =\exp (i k x),
\end{aligned}
$$

where $c \in \mathbb{R}$ is the constant wavespeed and the initial condition represents an isolated Fourier component. Such a choice for $u(x, 0)$ is motivated by the linearity of the problem. The solution for a general initial condition, $U_{0}(x)$, amenable to the Fourier Transform, $U_{0}(x)=\int_{-\infty}^{\infty} \hat{U}(k) \exp (i k x) \mathrm{d} k$ can be obtained by linear superposition of solutions of Eqn. (1). Moreover, support on the entire real line allows us to investigate the limit of asymptotic resolution $k \rightarrow 0$ in the subsequent sections.

For a general nodal discontinuous finite element scheme of polynomial order $P$, the domain is partitioned into non-overlapping elements, $\Omega=\bigcup_{n} \Omega_{n}$ where $\Omega_{n}=\left[x_{n}, x_{n+1}\right)$ and the sequence of nodes, $\left\{x_{n}\right\}$, is increasing on the real line. Each element is further discretized with $P+1$ variably distributed, distinct solution points $\boldsymbol{x}_{n}=\left[x_{n, 1}, x_{n, 2}, \ldots, x_{n, P+1}\right]^{T}$. We adopt the notation of indicating vectors using boldface symbols as well as sub-script indexing so that $\boldsymbol{x}_{n}=\left(x_{n, p}\right)_{p=1,2, \ldots, P+1}$. The numerical solution, $u^{\delta}(x, t)$, is defined as a piecewise interpolating polynomial such that in the $n^{\text {th }}$ element,

$$
u^{\delta}\left(x \in \Omega_{n}, t\right):=\sum_{p=1}^{P+1} u_{n, p}^{\delta}(t) \ell_{n, p}(x),
$$


where $\ell_{n, p}$ is the $p^{t h}$ Lagrange polynomial in the $n^{\text {th }}$ element. The solution is allowed to be discontinuous at the element interfaces. Assuming exact integration in time, Eqn. (1) leads to the semi-discrete numerical conservation law,

$$
\begin{aligned}
\frac{d \boldsymbol{u}_{n}^{\boldsymbol{\delta}}}{d t}+\left.c \frac{\partial^{\delta} \boldsymbol{u}^{\boldsymbol{\delta}}}{\partial x}\right|_{n} & =0, \quad \forall n, t>0 \\
\boldsymbol{u}_{n}^{\boldsymbol{\delta}}(0) & =\exp \left(i k \boldsymbol{x}_{n}\right)
\end{aligned}
$$

where $\boldsymbol{u}_{n}^{\boldsymbol{\delta}}(t)=\left(u_{n, p}^{\delta}(t)\right)_{p=1,2, \ldots, P+1}$ denotes the vector of solution values and $\left.\frac{\partial^{\delta} \boldsymbol{u}^{\delta}}{\partial x}\right|_{n}$ is the vector of numerically evaluated derivative values in the $n^{\text {th }}$ element, which constitutes the defining feature of the scheme.

Consider introducing a linear isoparametric map from the physical domain $x \in \Omega_{n}$ to the parent domain $\xi \in[-1,1)$ such that

$$
\left.\xi\right|_{\Omega_{n}}(x):=2 \frac{x-x_{n}}{x_{n+1}-x_{n}}-1
$$

We will restrict our attention to the case of uniform element size $h=x_{n+1}-$ $x_{n}, \forall n$ and identical distribution of solution points across elements in the parent space $\boldsymbol{\xi}=\left(\xi_{p}\right)_{p=1,2, \ldots, P+1}$. This restriction leads to a purely elementlocal description of the semi-discrete numerical conservation law which can be expressed as

$$
\begin{aligned}
\frac{d \boldsymbol{u}_{n}^{\boldsymbol{\delta}}}{d t}+c \frac{2}{h} \boldsymbol{Q}(k h) \boldsymbol{u}_{n}^{\boldsymbol{\delta}} & =0, \quad \forall n, t>0 \\
\boldsymbol{u}_{n}^{\boldsymbol{\delta}}(0) & =\exp \left(i k x_{n}\right) \boldsymbol{w}_{0}
\end{aligned}
$$

where $\boldsymbol{w}_{0}$ is the collocation projection of the initial condition onto the solution points,

$$
\boldsymbol{w}_{0}=\left(\exp \left(i k \frac{h}{2}\left(1+\xi_{p}\right)\right)\right)_{p=1,2, \ldots, P+1},
$$

and $\boldsymbol{Q}(k h) \in \mathbb{C}^{(P+1) \times(P+1)}$ is the numerical differentiation operator in the parent space, specific to the scheme. For instance, in the case of Flux Reconstruction $[11,23,37]$, the numerical derivative is defined by differentiating a piecewise polynomial of degree $P+1$ which is globally continuous on account 
of corrections emanating from the element interfaces,

$$
\begin{aligned}
c \frac{\partial^{\delta} u^{\delta}}{\partial x}(x, t):= & c \frac{\partial u^{\delta}}{\partial x}(x, t) \\
& +\frac{2}{h}\left(c u_{I}^{\delta}\left(x_{n}, t\right)-c u^{\delta}\left(x_{n}^{+}, t\right)\right) \frac{d g_{L}}{d \xi}(\xi(x)) \\
& +\frac{2}{h}\left(c u_{I}^{\delta}\left(x_{n+1}, t\right)-c u^{\delta}\left(x_{n+1}^{-}, t\right)\right) \frac{d g_{R}}{d \xi}(\xi(x)), \quad x \in \Omega_{n},
\end{aligned}
$$

where $c u_{I}^{\delta}\left(x_{n}\right)$ denotes the interface flux computed from the polynomial functions on either side of the interface,

$$
u_{I}^{\delta}\left(x_{n}\right):=(1-\alpha) u^{\delta}\left(x_{n}^{-}\right)+\alpha u^{\delta}\left(x_{n}^{+}\right),
$$

where $\alpha \in\left[0, \frac{1}{2}\right]$ denotes the upwinding coefficient, $(.)^{-},(.)^{+}$denote the left and right hand limits, $g_{L}(\xi), g_{R}(\xi) \in \mathbb{P}_{P+1}$ are the left-boundary and right-boundary correction functions in the parent space which satisfy the constraints,

$$
\begin{aligned}
& g_{L}(-1)=g_{R}(+1)=1 \\
& g_{L}(+1)=g_{R}(-1)=0 .
\end{aligned}
$$

A detailed description with schematics and motivation is provided in [11]. Plugging Eqn. (8) into Eqn. (7) and utilizing the exponential displacement relations, $u^{\delta}\left(x_{n}^{-}, t\right)=\exp (-i k h) u^{\delta}\left(x_{n+1}^{-}, t\right), u^{\delta}\left(x_{n+1}^{+}, t\right)=\exp (i k h) u^{\delta}\left(x_{n}^{+}, t\right), x \in$ $\Omega_{n}$, we get

$$
\begin{aligned}
c \frac{\partial^{\delta} u^{\delta}}{\partial x}(x, t)= & c \frac{\partial u^{\delta}}{\partial x}(x, t) \\
& +c \frac{2}{h}(1-\alpha)\left(\exp (-i k h) u^{\delta}\left(x_{n+1}^{-}, t\right)-u^{\delta}\left(x_{n}^{+}, t\right)\right) \frac{d g_{L}}{d \xi}(\xi(x)) \\
& +c \frac{2}{h} \alpha\left(\exp (i k h) u^{\delta}\left(x_{n}^{+}, t\right)-u^{\delta}\left(x_{n+1}^{-}, t\right)\right) \frac{d g_{R}}{d \xi}(\xi(x)), \quad x \in \Omega_{n},
\end{aligned}
$$

which can be expressed in the vector form of Eqn. (5) using Eqn. (3) and defining

$\boldsymbol{Q}(k h):=\boldsymbol{D}+(1-\alpha) \boldsymbol{g}_{\boldsymbol{L}, \boldsymbol{\xi}}\left(\exp (-i k h) \boldsymbol{\ell}_{+}^{T}-\boldsymbol{\ell}_{-}^{T}\right)+\alpha \boldsymbol{g}_{\boldsymbol{R}, \boldsymbol{\xi}}\left(\exp (i k h) \boldsymbol{\ell}_{-}^{T}-\boldsymbol{\ell}_{+}^{T}\right)$ 
Here $\boldsymbol{D} \in \mathbb{R}^{(P+1) \times(P+1)}$ is the polynomial differentiation operator such that

$$
D_{p, m}=\frac{\mathrm{d} \ell_{m}}{\mathrm{~d} \xi}\left(\xi_{p}\right), \quad p, m=1,2, \ldots, P+1,
$$

where $\ell_{m}$ is the $m^{\text {th }}$ Lagrange polynomial in the parent domain. $\boldsymbol{g}_{\boldsymbol{L}, \boldsymbol{\xi}}, \boldsymbol{g}_{\boldsymbol{R}, \boldsymbol{\xi}} \in$ $\mathbb{R}^{(P+1) \times 1}$ are the derivatives of the left-boundary and right-boundary correction functions at the solution points, and $\boldsymbol{\ell}_{-}, \boldsymbol{\ell}_{+} \in \mathbb{R}^{(P+1) \times 1}$ are the extrapolated values of the $P+1$ Lagrange basis polynomials,

$$
\ell_{+_{p}}=\ell_{p}(+1), \ell_{-p}=\ell_{p}(-1), \quad p=1,2, \ldots, P+1 .
$$

\subsection{Analytical solution of the exact and semi-discrete conservation laws}

The element-local semi-discrete numerical conservation law, Eqn. (5), represents a simple linear dynamical system that can be analytically integrated in time using matrix factorization. Assuming that the differentiation matrix is diagonalizable,

$$
\boldsymbol{Q}(k h)=\boldsymbol{W}(k h) \boldsymbol{\Gamma}(k h) \boldsymbol{W}^{-1}(k h)=\boldsymbol{W}(k h) i k \frac{h}{2} \boldsymbol{\Lambda}(k h) \boldsymbol{W}^{-1}(k h),
$$

where $\boldsymbol{\Gamma}$ is the diagonal matrix of eigenvalues $\gamma_{p}(k h) \in \mathbb{C}$ for $p=1,2, \ldots, P+$ $1, c \boldsymbol{\Lambda}$ is the diagonal matrix of numerical wavespeeds, $c \lambda_{p}(k h) \in \mathbb{C}$, and $\boldsymbol{W} \in$ $\mathbb{C}^{P+1 \times P+1}$ is the dense matrix containing eigenvectors of the differentiation operator. In the remainder of this paper, we shall refer to $\lambda$ as the normalized eigenvalue and $\gamma=i k \frac{h}{2} \lambda$ as the full eigenvalue. Note that for correction functions with non-trivial derivatives, i.e. $\boldsymbol{g}_{\boldsymbol{L}, \boldsymbol{\xi}} \neq \mathbf{0}$, the matrix $\boldsymbol{Q}$ is complex and, empirically, we have not encountered a case where the assumption of diagonalizability has failed to hold.

The initial condition can also be expanded in the basis of the eigenvectors,

$$
\boldsymbol{w}_{0}=\boldsymbol{W} \boldsymbol{\beta},
$$

where $\beta_{p} \in \mathbb{C}$ is the expansion coefficient along the $p^{\text {th }}$ column of $\boldsymbol{W}, \boldsymbol{w}_{p}(k h)$, for $p=1,2, \ldots, P+1$. The solution to Eqn. (5) can now be written as

$$
\begin{aligned}
\boldsymbol{u}_{n}^{\boldsymbol{\delta}}(t) & =\exp \left(-c t\left(\frac{2}{h} \boldsymbol{Q}(k h)\right)\right) \boldsymbol{u}_{n}^{\boldsymbol{\delta}}(0) \\
& =\boldsymbol{W} \exp (-i k c t \boldsymbol{\Lambda}) \boldsymbol{W}^{-1} \exp \left(i k x_{n}\right) \boldsymbol{W} \boldsymbol{\beta} \\
& =\exp \left(i k x_{n}\right) \boldsymbol{W} \exp (-i k c t \boldsymbol{\Lambda}) \boldsymbol{\beta} \\
& =\exp \left(i k x_{n}\right) \sum_{p=1}^{P+1} \exp \left(-i k c \lambda_{p} t\right) \beta_{p} \boldsymbol{w}_{p},
\end{aligned}
$$


which shows that the numerical solution is a superposition of $P+1$ eigenmodes along the eigenvectors of $\boldsymbol{Q}$ weighted by the expansion coefficients, $\beta_{p}$, of the initial condition. The time evolution of these modes is dictated by the normalized eigenvalues $\lambda_{p}$.

We can also write the analytical form of the exact solution on the vector of solution points, $\boldsymbol{x}_{n}=x_{n}+\frac{h}{2}(1+\boldsymbol{\xi})$, by sampling the functional form $\exp (i k(x-c t))$

$$
\begin{aligned}
\boldsymbol{u}_{n}(t) & =\exp \left(i k x_{n}\right) \exp (-i k c t) \boldsymbol{W} \boldsymbol{\beta} \\
& =\exp \left(i k x_{n}\right) \exp (-i k c t) \sum_{p=1}^{P+1} \beta_{p} \boldsymbol{w}_{p}
\end{aligned}
$$

\subsection{Absolute error and interpretation of eigenpairs}

\subsubsection{Stability, dispersion and dissipation}

The eigenvalues associated with the differentiation operator provide a direct measure of the dispersive, dissipative and stability properties of the numerical scheme $[37,31,36]$. This can be readily observed by evaluating the vector of pointwise error at the solution points in the $n^{\text {th }}$ element,

$$
\boldsymbol{e}_{n}(t):=\boldsymbol{u}_{n}^{\delta}(t)-\boldsymbol{u}_{n}(t)=\left(u_{n, p}^{\delta}(t)-u_{n, p}(t)\right)_{p=1,2, \ldots, P+1} .
$$

Substituting Eqns. (16) and (17) into Eqn. (18),

$$
\begin{aligned}
\boldsymbol{e}_{n}(t) & =\exp \left(i k x_{n}\right) \exp (-i k c t) \sum_{p=1}^{P+1}\left(\exp \left(-i k c\left(\lambda_{p}-1\right) t\right)-1\right) \beta_{p} \boldsymbol{w}_{p} \\
& =\exp \left(i k x_{n}\right) \exp (-i k c t) \sum_{p=1}^{P+1}\left[\exp \left(k c \lambda_{p}^{\mathrm{Im}} t\right) \exp \left(-i k c\left(\lambda_{p}^{\mathrm{Re}}-1\right) t\right)-1\right] \beta_{p} \boldsymbol{w}_{p},
\end{aligned}
$$

Eqn. (19) shows that the condition for pointwise stability [11] of the numerical solution can be prescribed as

$$
\lambda_{p}^{\operatorname{Im}}(k h) \leq 0 \text { for } p=1,2, \ldots, P+1,
$$

which must hold for $k \in\left[0, \frac{(P+1) \pi}{h}\right]$ where the upper bound corresponds to the Nyquist limit for the grid. 
For a given mode indexed by $p$, the deviation from unity of the real part of the normalized eigenvalue, $\lambda_{p}^{\mathrm{Re}}(k h)-1$, leads to numerical dispersion, i.e. the numerical solution propagates at an incorrect speed. The imaginary part of the normalized eigenvalue, $\lambda_{p}^{\operatorname{Im}}(k h)$, accounts for numerical dissipation, i.e. the numerical solution decays in amplitude with time for a stable, upwinded scheme, $\left(\alpha<\frac{1}{2}\right)$.

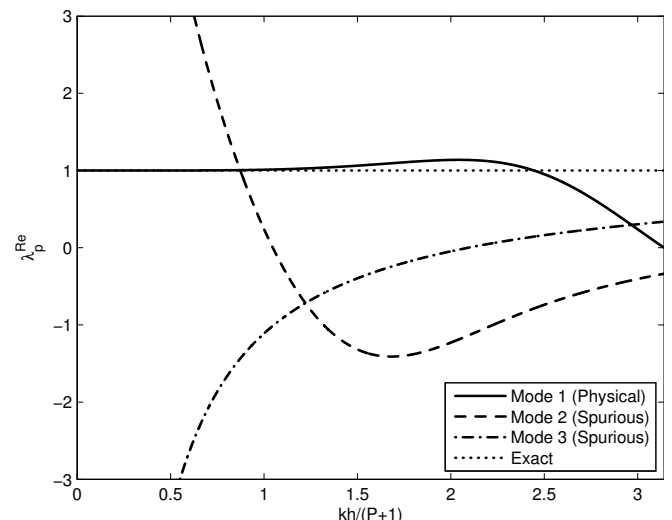

(a) $\lambda^{\mathrm{Re}}$ : measure of dispersion

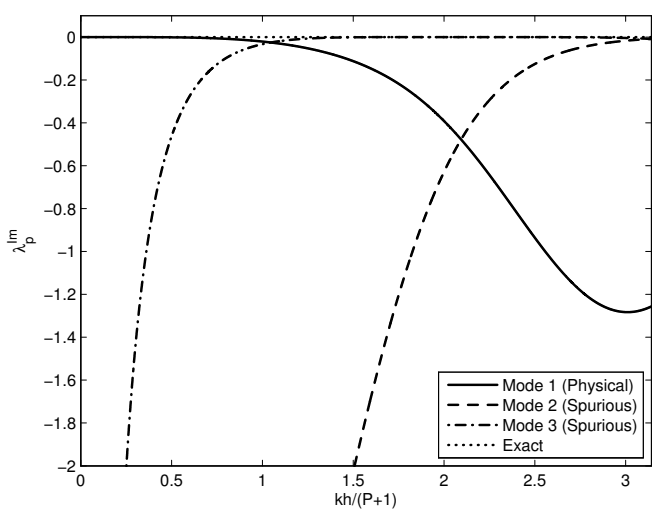

(b) $\lambda^{\mathrm{Im}}:$ measure of dissipation

Figure 1: Normalized eigenvalues: Real and imaginary parts of $\lambda_{p}$ for each of the numerical modes $p=1,2,3$ for the fully upwinded, $\alpha=0$, DG scheme of polynomial order $P=2$.

Figures 1 and 2 plot the real and imaginary parts of $\lambda$ for the three numerical modes in the case of the fully upwinded and centered DG schemes of polynomial order $P=2$. The plots suggest the definition of a 'physical' eigenmode, indexed at $p=1$, based on the error in the normalized eigenvalue in the asymptotic limit,

$$
\lim _{k h \rightarrow 0} \lambda_{1}(k h)=1
$$

All remaining modes, $p \neq 1$, exhibit excessive dispersion and dissipation errors as $k h \rightarrow 0$ and are therefore referred to as 'spurious' modes. The existence of a physical mode and the nature of the spurious modes is rigorously established in the upcoming sections. A recent exposition for the DG scheme can be found in [33]. Note that outside the asymptotic limit, the spurious modes may exhibit lower dispersion and/or dissipation errors than the physical mode, as can be clearly seen in Figure 2a. This motivates the investigation of how the initial condition is distributed among the $P+1$ eigenmodes as a function of $k h$, and is discussed in $\S 2.3 .2$. 


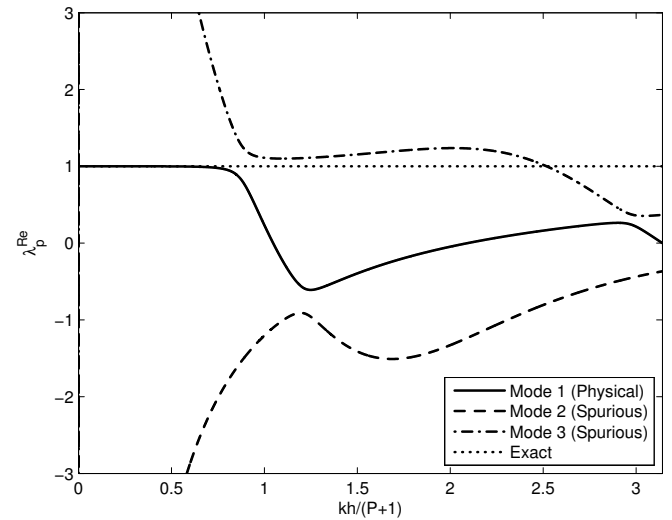

(a) $\lambda^{\mathrm{Re}}$ : measure of dispersion

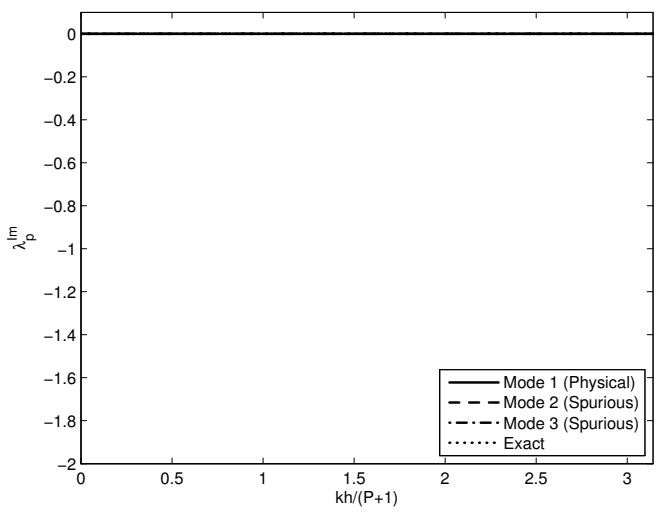

(b) $\lambda^{\mathrm{Im}}:$ measure of dissipation

Figure 2: Normalized eigenvalues: Real and imaginary parts of $\lambda_{p}$ for each of the numerical modes $p=1,2,3$ for the centered, $\alpha=0.5$, DG scheme of polynomial order $P=2$.

Note that in the case of a centered flux, $\alpha=\frac{1}{2}$, it can be shown that the numerical solution propagates without any dissipation, $\lambda_{p}^{\operatorname{Im}}=0 \forall p$, provided that the correction functions obey certain orthogonality conditions. In particular, Vincent et al. [23] have proven neutral stability in the weak sense that a certain broken Sobolev norm remains invariant with time when $\alpha=\frac{1}{2}$ and the correction functions belong to the so-called Energy-Stable FR family of functions.

\subsubsection{Modal weights and characteristic time scales of dissipation}

The eigenvectors dictate the distribution of energy among the physical and the spurious modes. For a given wavenumber $k$, the initial condition $\boldsymbol{w}_{0}$ is distributed among the $P+1$ numerical modes with weights given by $\boldsymbol{\beta}$. Figures $3 \mathrm{a}$ and $3 \mathrm{~b}$ plot $|\beta|^{2}$ for the 3 eigenmodes of the fully-upwinded and centered DG schemes of polynomial order $P=2$ using Gauss-Legendre solution points. We see that for low and moderate wavenumbers, the physical mode contains a dominant fraction of the energy. Moreover, in the limit of $k h \rightarrow 0$, the fraction of energy in the spurious modes vanishes. This aspect will also be rigorously proven in the next section.

For a stable and upwinded scheme, $\lambda_{p}^{\operatorname{Im}}<0$, we can define the half-life for each eigenmode,

$$
\tau_{p}=-\frac{1}{k c \lambda_{p}^{\operatorname{Im}}} \text { for } p=1,2, \ldots, P+1,
$$




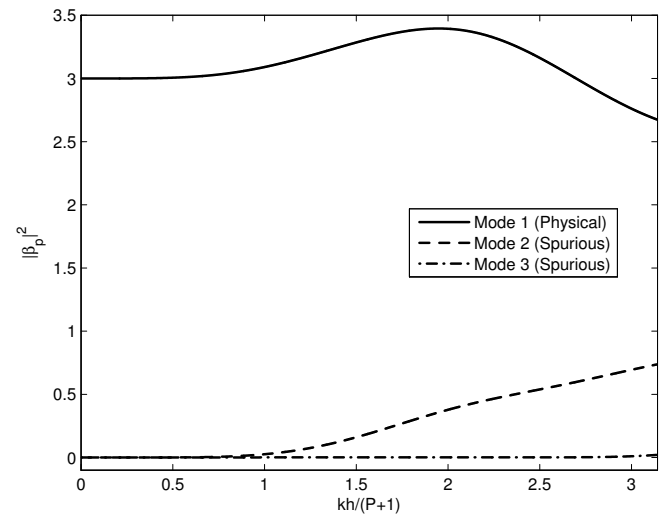

(a) Fully upwind interface flux, $\alpha=0$

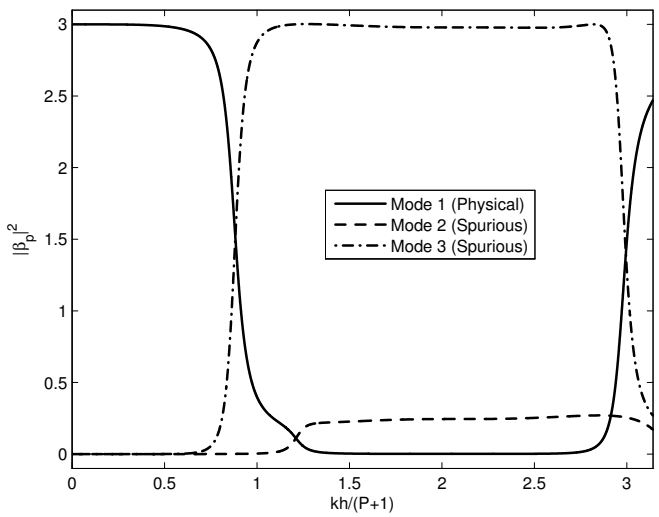

(b) Centered interface flux, $\alpha=\frac{1}{2}$

Figure 3: Energy distribution: $|\beta|^{2}$ for the 3 eigenmodes of the DG scheme of polynomial order $P=2$ using Gauss-Legendre solution points.

which is the time after which the amplitude of the mode has decayed by a factor of $\exp (-1)$. Table 1 records the half-lives for the 3 modes in the case of the fully upwinded DG scheme of polynomial order $P=2$ for $\frac{k h}{P+1}=$ $\frac{\pi}{6}$, unit grid spacing $h=1$ and unit wavespeed $c=1$. These parameter values correspond to a resolution of 12 solution points per wavelength of the sinusoidal initial condition. The tabulated values suggest that the numerical differentiation operator introduces two widely separated time scales,

$$
\tau_{\text {physical }}=\tau_{1} \gg \tau_{\text {spurious }}=\underset{p=2}{P+1} \max _{p}
$$

The energy $|\beta|^{2}$ associated with the spurious modes $p=2, \ldots, P+1$ is rapidly dissipated by an upwinded scheme within $\sim \tau_{\text {spurious }}$ time. The remaining energy is dissipated after a much longer time, $\tau_{\text {physical }}$. In the case of a centered flux, the energy associated with the spurious modes persists perpetually and such characteristic time scales cannot be defined.

\subsubsection{Evolution of pointwise error}

Numerical eigenmodes completely specify the evolution of pointwise error in time. To see this, consider the vector $\ell^{2}$ norm of pointwise error at the set of solution points in the $n^{\text {th }}$ element,

$$
\left\|\boldsymbol{e}_{n}(t)\right\|_{2}=\left\|\boldsymbol{u}_{n}^{\delta}(t)-\boldsymbol{u}_{n}(t)\right\|_{2}=\left[\sum_{p=1}^{P+1}\left|u_{n, p}^{\delta}(t)-u_{n, p}(t)\right|^{2}\right]^{1 / 2}
$$




\begin{tabular}{l|cccc|cc}
\hline & \multicolumn{4}{|c|}{$\alpha=0$} & \multicolumn{2}{c}{$\alpha=\frac{1}{2}$} \\
& $\lambda_{p}^{\mathrm{Re}}$ & $\lambda_{p}^{\mathrm{Im}}$ & $\tau_{p}$ & $\left|\beta_{p}\right|^{2}$ & $\lambda_{p}$ & $\left|\beta_{p}\right|^{2}$ \\
\hline Mode 1 (Physical) & 1.00032 & -0.00114 & 556.34096 & 3.00744 & 0.99874 & 2.98377 \\
Mode 2 (Spurious) & 4.13266 & -5.31987 & 0.11967 & 0.00065 & 4.41886 & 0.00058 \\
Mode 3 (Spurious) & -3.22312 & -0.40856 & 1.55820 & 0.00010 & -3.50774 & 0.00003 \\
Exact & 1 & 0 & - & - & 1 & - \\
\hline
\end{tabular}

Table 1: Normalized eigenvalues, half lives and energy distribution for the DG scheme of polynomial order $P=2$ for $\frac{k h}{P+1}=\frac{\pi}{6}$, unit grid spacing $h=1$ and unit wavespeed $c=1$.

This definition is motivated by the nodal nature of FR and is different from the usual functional norm that measures integrated error over a fixed domain. In particular, this simple Euclidean measure is local to the $n^{\text {th }}$ element and does not encounter any technical difficulties in the limit of $h \rightarrow 0$ which we will explore in the next section. Substituting Eqn. (19) in Eqn. (24),

$$
\begin{aligned}
\left\|\boldsymbol{e}_{n}(t)\right\|_{2} & =\left|\exp \left(i k x_{n}\right)\right||\exp (-i k c t)|\left\|\sum_{p=1}^{P+1}\left(\exp \left(-i k c\left(\lambda_{p}-1\right) t\right)-1\right) \beta_{p} \boldsymbol{w}_{p}\right\|_{2} \\
& =\left\|\sum_{p=1}^{P+1}\left(\exp \left(-i k c\left(\lambda_{p}-1\right) t\right)-1\right) \beta_{p} \boldsymbol{w}_{p}\right\|_{2}
\end{aligned}
$$

where we have used that $|\exp (\zeta)|=1$ if $\zeta$ is purely imaginary. Figure 4 shows the evolution of this norm against number of time periods, $T=$ $2 \pi /(k c)$, for the fully-upwinded DG scheme of polynomial order $P=2$ using Gauss-Legendre solution points, $\frac{k h}{P+1}=\frac{\pi}{6}$, unit grid spacing $h=1$ and unit wavespeed $c=1$ as in Table 1. For the upwinded scheme, the error grows rapidly within $\tau_{\text {spurious }}$ time owing to the loss of energy associated with the decay of spurious modes. This region is marked by oscillations associated with large phase errors. Beyond that time, the growth of error is much slower corresponding to the physical mode and is given by,

$$
\left\|\boldsymbol{e}_{n}\left(t \gg \tau_{\text {spurious }}\right)\right\|_{2} \simeq\left\|\left(\exp \left(-i k c\left(\lambda_{1}-1\right) t\right)-1\right) \beta_{1} \boldsymbol{w}_{1}+\left(\boldsymbol{w}_{0}-\beta_{1} \boldsymbol{w}_{1}\right)\right\|_{2}
$$

where $\boldsymbol{w}_{0}-\beta_{1} \boldsymbol{w}_{1}$ is the constant error introduced due to loss of energy in the spurious modes. On the other hand, in the case of a centered flux, the spurious modes do not decay at all and continue to contribute phase errors to the portion of the energy received by them from the initial condition. This 


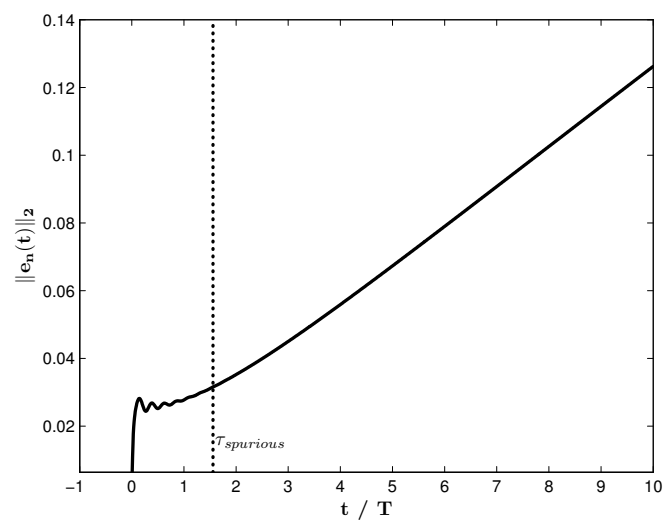

(a) Fully upwind interface flux, $\alpha=0$ of FR schemes.

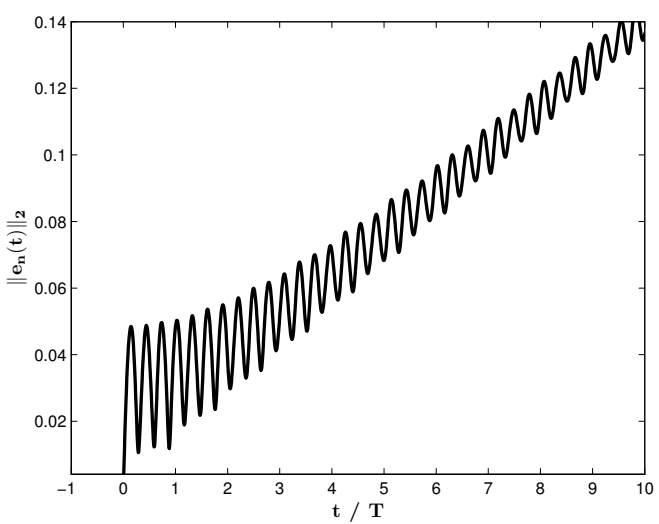

(b) Centered interface flux, $\alpha=\frac{1}{2}$

Figure 4: Evolution of the norm of pointwise error, Eqn. (25), against number of time periods, $T=2 \pi /(k c)$, for the DG scheme of polynomial order $P=2$ using Gauss-Legendre solution points, $\frac{k h}{P+1}=\frac{\pi}{6}$, unit grid spacing $h=1$ and unit wavespeed $c=1$.

again highlights why there is a prevalence of upwinded fluxes in applications

\section{Consistency and convergence of the FR formulation}

This section proves that any stable scheme within the Flux Reconstruction formulation, having symmetric correction functions, is consistent with respect to the linear advection problem and converges to the exact solution in the asymptotic limit, $k h \rightarrow 0$. The bodies of all proofs have been deferred to Appendix A to enhance readability.

\subsection{Eigenvalue decomposition of the polynomial differentiation matrix $\boldsymbol{D}$}

We begin by assembling results on the eigen-decomposition of the polynomial differentiation matrix which will be needed in the next subsection.

Lemma 3.1. Let $\boldsymbol{v} \in \mathbb{C}^{(P+1) \times 1}$ be the vector obtained from sampling a polynomial $v$ of degree $\leq P$ at $P+1$ distinct points, $\boldsymbol{\xi}=\left(\xi_{p}\right)_{p=1,2, \ldots, P+1}$, in $[-1,1]$. Then, the polynomial differentiation matrix $\boldsymbol{D}$ in Eqn. (12) exactly differentiates $\boldsymbol{v}$ to yield the vector obtained from sampling $\frac{d v}{d \xi}$ at the $P+1$ points. 
Lemma 3.2. For an FR scheme of polynomial order $P$ with distinct solution points, the polynomial differentiation matrix $\boldsymbol{D}$ in Eqn. (12) has $P+1$ repeated eigenvalues at 0 corresponding to the uni-dimensional eigenspace spanned by $\mathbf{1}=[1,1, \ldots, 1]^{T}$.

\subsection{Asymptotic eigenvalue decomposition of the differentiation matrix $\boldsymbol{Q}(k h)$}

A proof of convergence for stable Flux Reconstruction schemes can be constructed by showing that the numerical conservation law, Eqn. (3), is consistent, and that the pointwise error, Eqn. (19), vanishes in the asymptotic limit of grid resolution or wavenumber. Note that for a given choice of correction function and upwinding coefficient, the only remaining parameter is the non-dimensional wavenumber $k h$. The asymptotic limit of $k h \rightarrow 0$ can be achieved by decreasing the grid spacing $h$ for a given wavenumber $k$ or by decreasing $k$ for a given $h[11,36,34]$.

The exact conservation law, Eqn. (1), utilizes the exact differentiation operator $\frac{\partial}{\partial \xi}=\frac{h}{2} \frac{\partial}{\partial x}$ which is associated with the eigenvalue $i k \frac{h}{2} \rightarrow 0$ as $k h \rightarrow 0$. The proof of convergence for the FR formulation is complicated by the observation that the numerical eigenvalues for the spurious modes, $\left(\gamma_{p}=i k \frac{h}{2} \lambda_{p}\right)_{p \neq 1}$, do not converge to zero, i.e. they are inconsistent. This can be verified directly from the plots in [31, 37] or indirectly by noting the normalized eigenvalues in Figs. 1, 2 diverge as $k h \rightarrow 0$. However, it can be shown that when the distribution of weights among the modes, $\boldsymbol{\beta}$, is taken into account, the inconsistent spurious modes do not receive any energy in the asymptotic limit. Moreover, under suitable conditions, the physical eigenvalue $\gamma_{1}$ converges to $i k \frac{h}{2}$ as well. Consequently, the pointwise error does indeed vanish as required.

The results that follow are directed at an FR scheme of finite polynomial order $P$ having $P+1$ distinct solution points. We begin by showing that, although the normalized eigenvalues $\lambda_{p}$ may become unbounded, the full eigenvalues, $\gamma_{p}$ for $p=1,2, \ldots, P+1$, always remain bounded.

Lemma 3.3. All eigenvalues of the FR differentiation operator $\boldsymbol{Q}(k h)$ remain bounded.

Table 2 shows that the limits of the eigenvalues $\gamma_{p}(k h \rightarrow 0)$ are indeed bounded, for all the $P+1$ modes. The next three results describe the asymptotic behavior of the physical eigenvalue $\gamma_{1}$ and also $\lambda_{1}$, the physical eigenvector $\boldsymbol{w}_{1}$, and the modal weight vector $\boldsymbol{\beta}$. 


\begin{tabular}{lccc}
\hline & \multicolumn{2}{c}{$\alpha=0$} & $\alpha=\frac{1}{2}$ \\
& $\gamma_{p}^{\mathrm{Re}}$ & $\gamma_{p}^{\mathrm{Im}}$ & $\gamma_{p}$ \\
\hline Mode 1 (Physical) & 0.00000 & 0.00000 & 0.00000 \\
Mode 2 (Spurious) & 1.50000 & 3.57071 & $3.87298 i$ \\
Mode 3 (Spurious) & 1.50000 & -3.57071 & $-3.87298 i$ \\
Exact & 0 & 0 & 0 \\
\hline
\end{tabular}

Table 2: Full eigenvalues for the DG scheme of polynomial order $P=2$ for $\frac{k h}{P+1}=0$.

Lemma 3.4. In the limit $k h \rightarrow 0$, the $F R$ differentiation operator $\boldsymbol{Q}$ has at least one eigenpair which corresponds to the physical mode, $\gamma_{1} \rightarrow 0$ and $\boldsymbol{w}_{1} \rightarrow \frac{1}{\sqrt{P+1}} \mathbf{1}$

Lemma 3.5. In the limit $k h \rightarrow 0$, if the differentiation operator $\boldsymbol{Q}$ is diagonalizable, then the modal weights $\beta_{p}$, in Eqn. (15), converge to $\sqrt{P+1}\left(\delta_{p 1}\right)$ for $p=1,2, \ldots, P+1$, where $\delta$ is the Kronecker delta function.

The convergence of $\boldsymbol{\beta}$ can also be easily verified from Fig. 3 which shows that $\left|\beta_{1}\right|^{2} \rightarrow P+1$ and $\left|\beta_{p \neq 1}\right|^{2} \rightarrow 0$.

Lemma 3.6. In the limit $k h \rightarrow 0$, if the differentiation operator $\boldsymbol{Q}$ is diagonalizable, the normalized eigenvalue corresponding to the physical mode converges to unity, $\lambda_{1} \rightarrow 1$.

The convergence of the physical eigenvalue can be easily verified from Figs. 1 and 2 which show that $\lambda_{1} \rightarrow 1$ as $k h \rightarrow 0$. The consistency of the Flux Reconstruction formulation can now be proven as follows.

Theorem 3.1. The FR numerical conservation law, Eqn. (3), is consistent if the differentiation operator $\boldsymbol{Q}$ is diagonalizable.

We are now prepared to state the result on the convergence of the FR formulation.

Theorem 3.2. For any stable FR scheme, if the differentiation operator $\boldsymbol{Q}$ is diagonalizable, then as $k h \rightarrow 0$, the pointwise error vanishes, $\boldsymbol{e}_{n} \rightarrow \mathbf{0}$, guaranteeing convergence at the solution points. 
Note that the use of a spatially discrete numerical conservation law, Eqn. (5), has restricted us to pointwise convergence, i.e. the numerical solution converges to the exact solution at the set of solution points. Such results have been routinely used for Finite Difference schemes. However, it is easy to show that pointwise convegence holds for all points in the element owing to the smoothness of the exact and numerical solutions.

Lemma 3.7. Under the conditions of Theorem 3.2, an FR scheme which converges pointwise at the set of solution points, converges at all points in the element $\Omega_{n}$.

\section{Rate of convergence of the FR formulation}

This section shows that, in the asymptotic limit, pointwise error is bounded by a linear function of time. Here, the constant term depends on the rates of convergence of the modal weights, $\beta_{p}$, for the spurious modes, and linear term depends on the rate of convergence of the normalized physical eigenvalue, $\lambda_{1}$.

For short times, $t \sim \tau_{\text {spurious }}$, the rate of convergence is largely dictated by that of the spurious modes and is typically $\mathcal{O}\left((k h)^{P+1}\right)$. Thereafter, the growth of error is slow corresponding to the physical mode which can be superconvergent. Once the error associated with the physical mode becomes significantly greater than the fraction of energy received by the spurious modes, the scheme exhibits superconvergence. 


\subsection{Rate of convergence of pointwise error}

Theorem 4.1. For a convergent FR scheme of polynomial order $P$, the norm of pointwise error in the nth element, Eqn. (25), is bounded above by a term that grows linearly in time,

$$
\left\|\boldsymbol{e}_{n}(t)\right\|_{2} \leq 2 C_{2}(k h)^{\substack{P+1 \\ \text { min }}} q_{p}^{\beta}
$$

where $q_{1}^{\lambda}>0$ is the asymptotic rate of convergence of the normalized physical eigenvalue, and $q_{p}^{\beta}>0$ is that for the modal weights,

$$
\begin{aligned}
\left|\lambda_{1}(k h)-1\right| & \leq C_{1}(k h)^{q_{1}^{\lambda}}, \\
\left|\beta_{p}(k h)-\sqrt{P+1} \delta_{1 p}\right| & \leq C_{2}(k h)^{q_{p}^{\beta}},
\end{aligned}
$$

for $p=1,2, \ldots, P+1$ as $k h \rightarrow 0$.

Eqn. (27) shows that the rate of convergence for FR is bounded by the sum of two terms. The first term depends on the spurious modes and is constant with respect to time. The second term depends on the physical mode and grows linearly in time. For short times, $t \sim \tau_{\text {spurious }}$, the order of convergence is bounded by $\min _{p=2}^{P+1} q_{p}^{\beta}$ corresponding to the rate at which the modal weights for the spurious modes vanish. After a long time, $t \gg \tau_{\text {spurious }}$, the order of convergence is bounded by $q_{1}^{\lambda}$ corresponding to the rate at which the normalized physical eigenvalue approaches the exact value. The linearity of the long-time error can be clearly observed in Fig. 4.

It is important to note that the long-time convergence rate in Eqn. (27) is sensitive to the process through which the limit of $k h \rightarrow 0$ is realized. If $k \rightarrow 0$ for constant $h$, the order is one unit higher than if $h \rightarrow 0$ for constant $k$ since

$$
\sqrt{P+1} c k(k h)^{q_{1}^{\lambda}} t=\sqrt{P+1} c k^{q_{1}^{\lambda}+1} h^{q_{1}^{\lambda}} t .
$$

This difference of one order can be observed in the definitions of the 'order of accuracy' proposed, somewhat heuristically, in [11,36]. It is clear now that those definitions correspond to the long-time rate obtained when $h \rightarrow 0$ 
for constant $k$. Such long-time super-convergence is realized for problems exhibiting an eventual quasi-steady solution which does not depend on the initial condition [42]. However, for most other practical simulations, the realized convergence rate would typically lie somewhere in between the shorttime and long-time rates depending on the characteristic time-scale of the problem.

\begin{tabular}{lcccc}
\hline & \multicolumn{2}{c}{$\alpha=0$} & \multicolumn{2}{c}{$\alpha=\frac{1}{2}$} \\
& $q_{p}^{\lambda}$ & $q_{p}^{\beta}$ & $q_{p}^{\lambda}$ & $q_{p}^{\beta}$ \\
\hline Mode 1 (Physical) & 4.998 & 4.002 & 6.004 & 4.004 \\
Mode 2 (Spurious) & - & 3.037 & - & 4.057 \\
Mode 3 (Spurious) & - & 2.956 & - & 3.944
\end{tabular}

Table 3: Rates of convergence for the physical and the spurious modes for the DG scheme of polynomial order $P=2$ on Gauss Legendre solution points. The rates are calculated using $\frac{k h}{(P+1) \pi}=0.01$ and $\frac{k h}{(P+1) \pi}=0.02$.

Table 3 records the relevant rates of convergence, $q_{p}^{\lambda}, q_{p}^{\beta}$, for the physical and the spurious modes for the fully upwinded and centered DG schemes of polynomial order $P=2$ on Gauss Legendre solution points. In the case of $\alpha=0$, we expect the rate of convergence to start from $\min _{p=2}^{3} q_{p}^{\beta} \simeq 3$ and asymptotically approach $q_{1}^{\lambda} \simeq 5$, when $h$ is refined for fixed $k$. This behavior can be verified from Fig. 5a which plots the evolution of the rate of convergence for the fully upwinded DG scheme of polynomial order $P=2$ on Gauss Legendre solution points, $k=\frac{\pi}{2}$, unit wavespeed $c=1$, using a pair of grid spacings $h=\{0.5,0.25\}$. Similarly, when refining $k$ for fixed $h$, on account of Eqn. (30), we expect the convergence rate to start from $\sim 3$ and asymptote to $\sim 6$. This behavior can be verified from Fig. $5 \mathrm{~b}$ which plots the convergence rate for grid spacing $h=0.25$ and two wavenumbers $k=\left\{\pi, \frac{\pi}{2}\right\}$.

In the case of a centered flux, the absence of dissipation implies that the spurious modes persist forever. The phase error introduced by these modes manifests in the oscillatory rate of convergence that can be seen in Figs. 6a and $6 \mathrm{~b}$. The superconvergent rate is observed only after the error in the physical mode grows to dominate over that of the spurious modes.

Theorem 4.1 provides a semi-analytical procedure for evaluating both the short-time and the long-time rate of convergence for any given scheme within the FR formulation. This procedure involves a direct eigenvalue computation 


\begin{tabular}{c|cc|cc}
\hline & \multicolumn{2}{|c}{$\alpha=0$} & \multicolumn{3}{c}{$\alpha=\frac{1}{2}$} \\
$\mathrm{P}$ & $q_{1}^{\lambda}$ & $\min _{p=2} q_{p}^{\beta}$ & $q_{1}^{\lambda}$ & $\begin{array}{c}P+1 \\
\min _{p=2}\end{array} q_{p}^{\beta}$ \\
\hline 0 & 1 & - & 2 & - \\
1 & 3 & 2 & 2 & 1 \\
2 & 5 & 3 & 6 & 4 \\
3 & 7 & 4 & 6 & 3 \\
4 & 9 & 5 & 10 & 6 \\
5 & 11 & 6 & 10 & 5 \\
\hline$P$ & $2 P+1$ & $P+1$ & $\begin{cases}2 P+2, & \text { even } P \\
2 P, & \text { odd } P\end{cases}$ & $\begin{cases}P+2, & \text { even } P \\
P, & \text { odd } P\end{cases}$
\end{tabular}

Table 4: Rates of convergence of the eigensystem components for DG using GaussLegendre solution points obtained from direct eigenvalue computation and rounded to nearest integer values. 


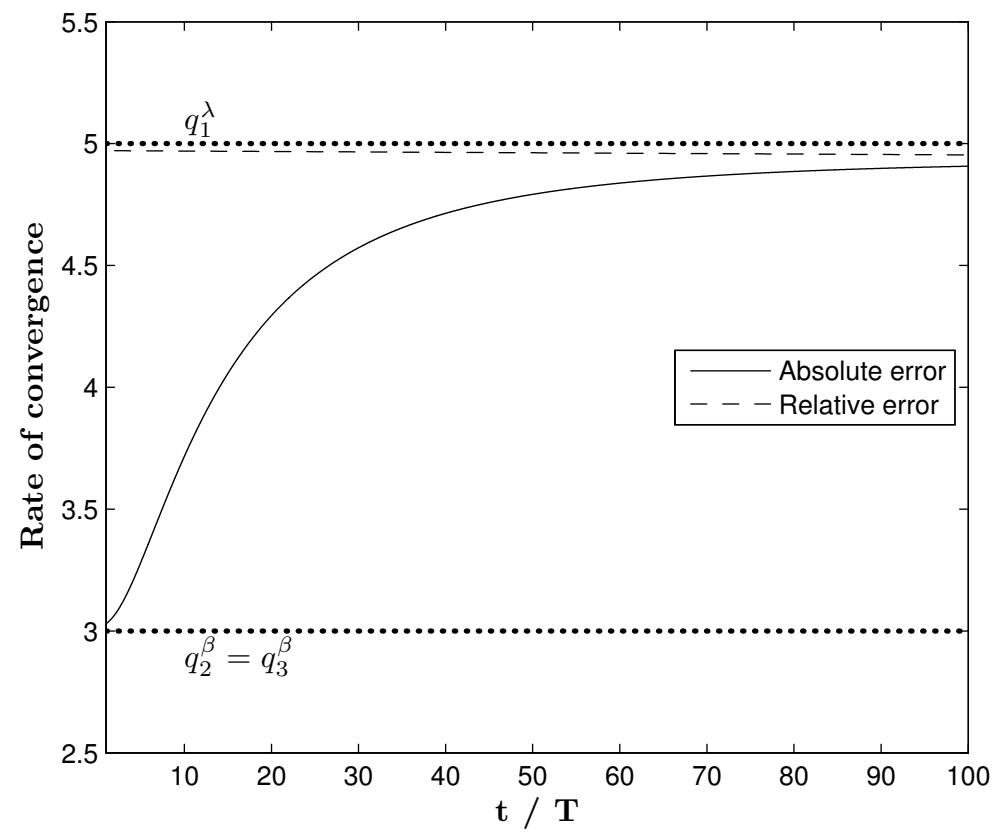

(a) Refinement in grid spacing: $k=\frac{\pi}{2}$, grid spacings $h=\{0.5,0.25\}$.

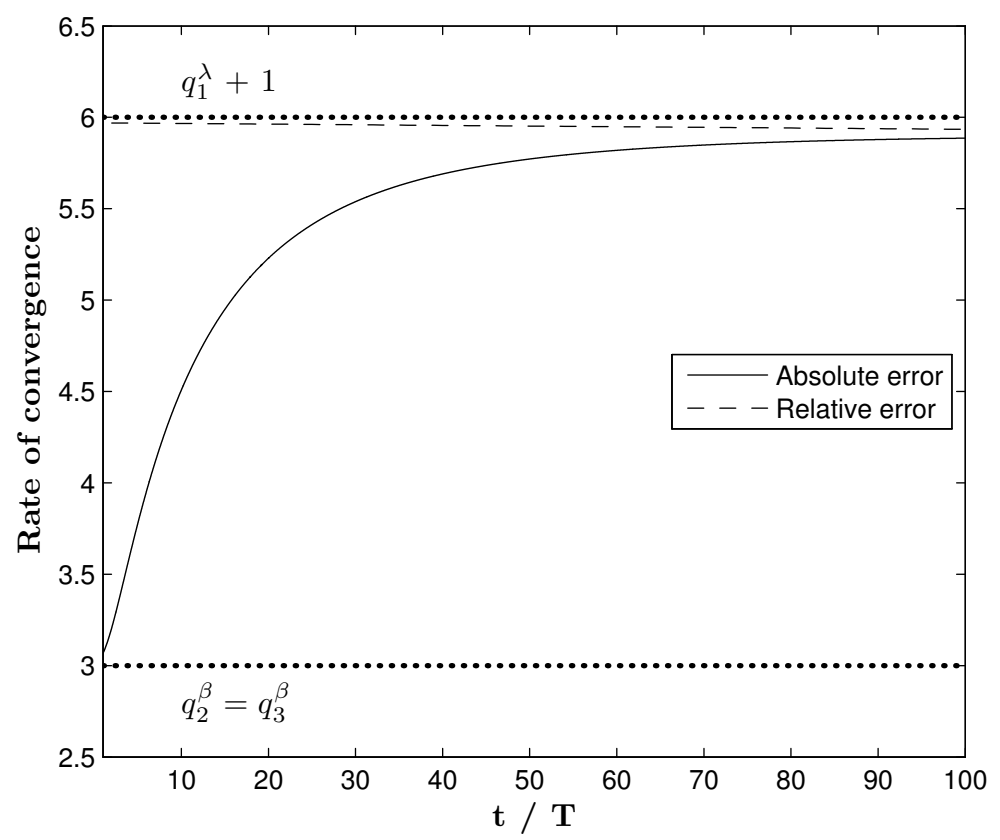

(b) Refinement in wavenumber: $h=0.25$, wavenumbers $k=\left\{\pi, \frac{\pi}{2}\right\}$.

Figure 5: Rate of convergence for the fully upwinded DG scheme of polynomial order $P=2$ on Gauss Legendre solution points with unit wavespeed $c=1$. The reference time for relative error is taken to be $t_{0}=T$. 


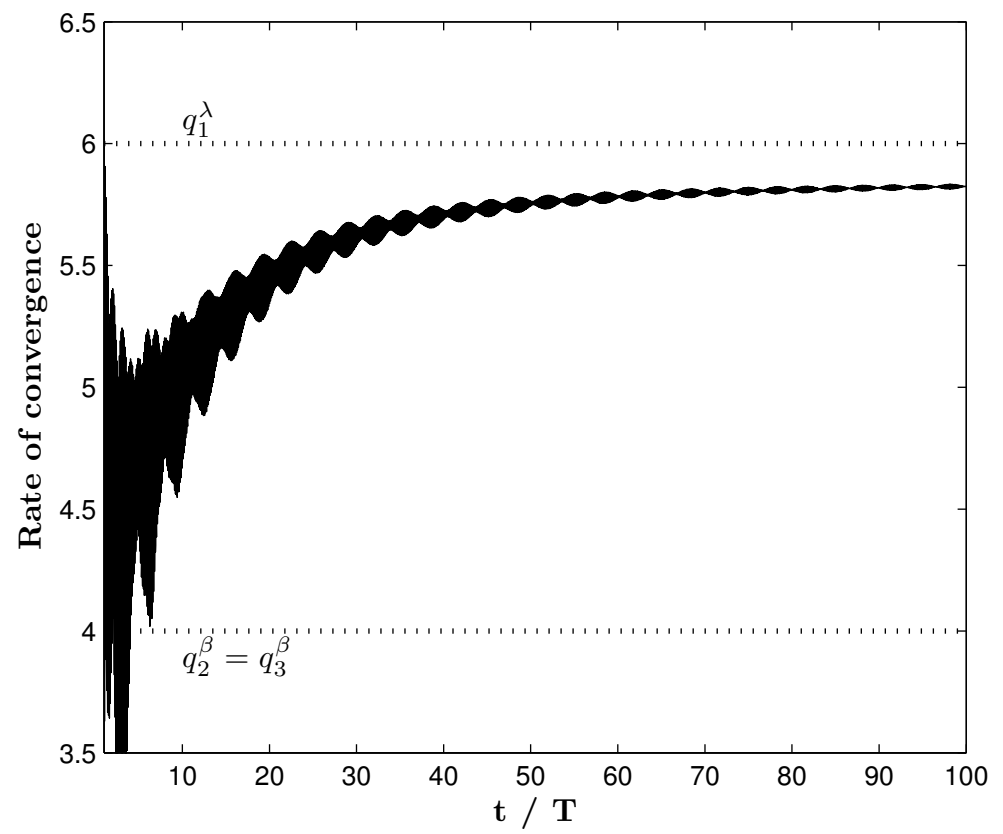

(a) Refinement in grid spacing: $k=\frac{\pi}{2}$, grid spacings $h=\{0.5,0.25\}$.

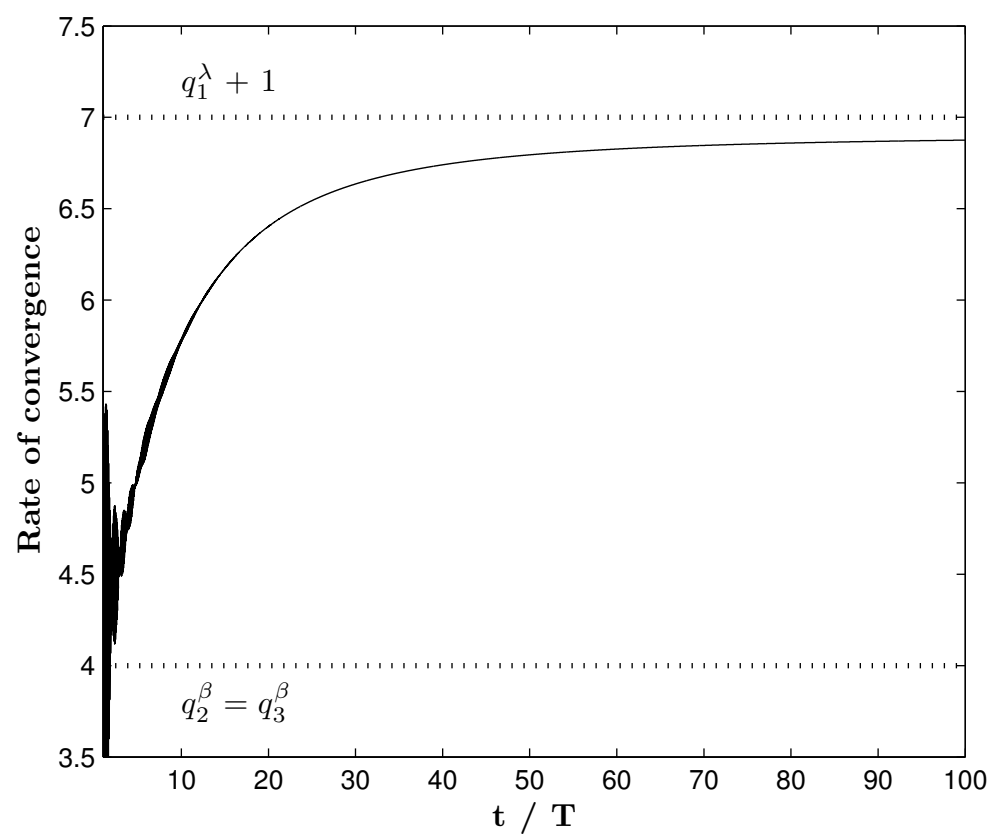

(b) Refinement in wavenumber: $h=0.25$, wavenumbers $k=\left\{\pi, \frac{\pi}{2}\right\}$.

Figure 6: Rate of convergence for the centered DG scheme of polynomial order $P=2$ on Gauss Legendre solution points with unit wavespeed $c=1$. 
which requires almost no computational effort (instantaneous on modern machines for all practical values of $P$ ). This is a significant improvement over grid-convergence studies of the form used until very recently [36] which can potentially require multiple days of computing time for determining the long-time rate of convergence.

Table 4 records the short-time and long-time rates of convergence for $P=0$ to 5 for the DG scheme using Gauss-Legendre solution points for both upwinded and centered fluxes. We see that, for a given polynomial order $P$, the short-time rate of convergence is given by $P+q$ and the long-time rate by $2 P+q$ where $q \in\{0,1,2\}$ depends on the type of interface flux and whether $P$ is odd or even. These results for DG agree well with Proposition 3.5 in [40] wherein Guo et al. derived the closed-form counterpart of Eqn. (27) for the upwinded DG scheme with $P=1,2,3$ using symbolic computations in Mathematica. Similarly, in comparison to the superconvergence studies by Cheng and Shu $[38,39]$ which established a lower bound of $P+3 / 2$ for the long-time rate of the upwinded DG scheme, these results provide a tighter bound of $2 P+1$ in the case of uniform meshes. This tightness can be observed in the numerical experiments reported in this paper as well as those in $[38,36]$. Note, however, that the extension of Theorem 4.1 to non-uniform meshes is not straightforward and requires alternatives to Fourier analysis as demonstrated in [39].

\subsection{Rate of convergence of 'relative' error for upwinded schemes}

From a computational perspective, using a conventional grid convergence study to determine the rate of convergence of the physical mode requires longtime integration while ensuring that the time step is appropriately small to minimize time-stepping errors. This results in an intensive exercise even for simple 1-D cases. Similar to the discourse in [40], we show that the wavepropagation error defined in $[29,36]$ allows the long-time rate to be deduced with minimal computational effort in the case of upwinded schemes. Towards this end, define a 'relative' error of the form,

$$
\boldsymbol{e}_{n}^{R}\left(t_{0}+m T ; t_{0}\right):=\boldsymbol{u}_{n}^{\boldsymbol{\delta}}\left(t_{0}+m T\right)-\boldsymbol{u}_{n}^{\boldsymbol{\delta}}\left(t_{0}\right),
$$

where $T=2 \pi /(k c)$ is the time period of the wave, $t_{0}$ is a reference time and $m \in \mathbb{Z}^{+}$is the number of time periods since the reference time. Such a definition suppresses the error offset introduced due to the spurious modes 
outlined in Eqn. (26),

$$
\begin{aligned}
\left\|\boldsymbol{e}_{n}^{R}\left(t_{0} \gg \tau_{\text {spurious }}\right)\right\|_{2} & =\left\|\boldsymbol{e}_{n}\left(t_{0}+m T\right)-\boldsymbol{e}_{n}\left(t_{0}\right)\right\|_{2} \\
& \left.\simeq \| \exp \left(-i k c\left(\lambda_{1}-1\right)\left(t_{0}+m T\right)\right)-\exp \left(-i k c\left(\lambda_{1}-1\right) t_{0}\right)\right] \beta_{1} \boldsymbol{w}_{1} \|_{2} \\
& =\left|\exp \left(-i k c\left(\lambda_{1}-1\right) t_{0}\right)\left(\exp \left(-i k c\left(\lambda_{1}-1\right) m T\right)-1\right)\right|\left|\beta_{1}\right| \\
& \leq\left|\exp \left(-i k c\left(\lambda_{1}-1\right) m T\right)-1\right|\left|\sqrt{P+1}+\mathcal{O}\left((k h)^{q_{1}^{\beta}}\right)\right| \\
& =\left|-i k c\left(\lambda_{1}-1\right) m T+\mathcal{O}\left(k^{2}\left(\lambda_{1}-1\right)^{2}\right)\right| \sqrt{P+1} \\
& =\sqrt{P+1} c k \mathcal{O}\left((k h)^{q_{1}^{\lambda}}\right) m T,
\end{aligned}
$$

which shows that the rate of convergence of the relative error is bounded only by the rate of convergence of the normalized eigenvalue $\lambda_{1}$. As can be seen from Table 1 , typically $\tau_{\text {spurious }}<T$, so that a choice of $t_{0}=T$ is sufficient. This can be verified from Fig. 5 which shows that the rate of convergence computed for the relative error yields the value $\sim 5$, for $h$ refinement, and $\sim 6$, for $k$ refinement, for all $m \geq 1$.

\section{Numerical experiments in 2-D}

In this final section, we perform additional numerical experiments to investigate if the theoretical rates of convergence derived for 1-D linear advection also hold for more general conservation laws. The first experiment introduces diffusion along with advection for a scalar over a 2-D domain discretized uniformly with quadrilaterals. Details regarding the application of FR schemes to such second-order equations can be found in [43]. The second experiment deals with a system of non-linear equations in 2-D subjected to a special initial condition at $t=0$ that allows for a smooth analytical solution for all time $t \geq 0$. The results are plotted in the style of Fig. 5 .

For generality of interpretation, we measure error with respect to both discrete and functional standard norms. The discrete $\ell^{2}\left(\Omega^{\delta}\right)$ norm is simply the averaged vector norm of pointwise error at the set of solution points,

$$
\|e\|_{\ell^{2}\left(\Omega^{\delta}\right)}(t):=\left[\frac{1}{N(P+1)^{n_{\mathrm{sd}}}} \sum_{n=1}^{N} \sum_{p=1}^{(P+1)^{n_{\mathrm{sd}}}}\left|e\left(x_{n, p}, t\right)\right|^{2}\right]^{1 / 2}
$$

where $x_{n, p}$ is the $p$ th solution point in the $n$th element, $N$ is the total number of elements, and $n_{\text {sd }}$ is the number of space-dimensions. The functional $L^{2}(\Omega)$ 
norm is the standard 2-norm of error over the entire domain,

$$
\|e\|_{L^{2}(\Omega)}(t):=\left[\int_{\Omega}|e(x, t)|^{2} \mathrm{~d} \Omega\right]^{1 / 2} .
$$

Note that Theorem 4.1 prescribes the rate of convergence only at the set of solution points, i.e. with respect to the $\ell^{2}\left(\Omega^{\delta}\right)$ norm. However, the rate of convergence at any other point in the element can at the least be easily bounded. Towards this end, use Eqn. (A.23) in the proof of Lemma 3.7, so that for $x \in \Omega_{n}$,

$$
\begin{aligned}
|e(x, t)| & \leq\left|\sum_{p=1}^{P+1}\left(u_{n, p}^{\delta}(t)-u_{n, p}(t)\right) \ell_{n, p}(x)\right|+|a(P, \boldsymbol{\xi})|(k h)^{P+1} \\
& \leq \sum_{p=1}^{P+1}\left|u_{n, p}^{\delta}(t)-u_{n, p}(t)\right|\left|\ell_{n, p}(x)\right|+|a(P, \boldsymbol{\xi})|(k h)^{P+1} \\
& \leq \max _{p=1}^{P+1}\left|\ell_{n, p}(x)\right|\left\|\boldsymbol{e}_{n}(t)\right\|_{1}+|a(P, \boldsymbol{\xi})|(k h)^{P+1} \\
& \leq \mu \max _{p=1}^{P+1}\left|\ell_{n, p}(x)\right|\left\|\boldsymbol{e}_{n}(t)\right\|_{2}+|a(P, \boldsymbol{\xi})|(k h)^{P+1} \\
& \leq \mu \max _{p=1}^{P+1}\left|\ell_{n, p}(x)\right|\left[2 C_{2}(k h)^{P+1} \min _{p=2}^{\beta}+C_{1} \sqrt{P+1} c k(k h)^{q_{1}^{\lambda}} t\right]+|a(P, \boldsymbol{\xi})|(k h)^{P+1},
\end{aligned}
$$
$\mathbb{R}$

$$
\begin{aligned}
\frac{\partial u}{\partial t}+a_{x} \frac{\partial u}{\partial x}+a_{y} \frac{\partial u}{\partial y}-b\left(\frac{\partial^{2} u}{\partial x^{2}}+\frac{\partial^{2} u}{\partial y^{2}}\right) & =0, \quad(x, y) \in(-1,1)^{2}, t>0, \\
u(x, y, 0) & =\sin (\pi x) \sin (\pi y),
\end{aligned}
$$


where $a_{x} \in \mathbb{R}, a_{y} \in \mathbb{R}$ are the advection speeds, and $b \in \mathbb{R}_{+}$is the constant diffusion coefficient. The boundary conditions are assumed to be periodic for simplicity. The analytical solution to Eqns. (36 - 37) for periodic boundary conditions is given by

$$
u(x, y, t)=\exp \left(-2 b \pi^{2} t\right) \sin \left(\pi\left(x-a_{x} t\right)\right) \sin \left(\pi\left(y-a_{y} t\right)\right), \quad x \in[-1,1]^{2}, t \geq 0 .
$$

This problem is solved using DG, recovered via FR, for $P=2,3$ using GaussLegendre points as solution points. The numerical solution is marched in time using the standard four-stage fourth order accurate RK4 time-stepping scheme for two thousand time-periods in order to capture the long-time rate of convergence. At the same time, the time-step is chosen small enough to ensure that errors due to numerical integration are much smaller than those due to spatial-discretization. Specifically, the time step is fixed at $10^{-3}$ for $P=2$ and $10^{-4}$ for $P=3$. The longest calculation involves roughly 40 million iterations. The advection speeds are set to unity, $a_{x}=a_{y}=$ 1. The diffusion coefficients are chosen small enough to ensure that the amplitude of the solution remains much larger than machine precision for the entire calculation. Specifically, $b=10^{-3}$ for $P=2$ and $10^{-4}$ for $P=3$. The common interface value for the advective flux is calculated using the Lax-Friedrichs or Rusanov formula (parameterized by upwinding parameter $\alpha$, Eqn. (8)), while that for the diffusive flux is calculated using the LDG formula (parameterized by upwinding coefficient $\beta_{\mathrm{LDG}}$, and jump coefficient $\left.\tau_{\mathrm{LDG}}\right)$. Both one-sided, isotropic $\left(\alpha=0, \beta_{\mathrm{LDG}}=1 / 2, \tau_{\mathrm{LDG}}=0\right)$ and fully centered $\left(\alpha=1 / 2, \beta_{\mathrm{LDG}}=0, \tau_{\mathrm{LDG}}=0\right)$ fluxes are investigated. The rate of convergence is calculated at each time-step using two uniform grids with $8 \times 8$ and $16 \times 16$ elements respectively.

Figures 7 and 8 plot the rate of convergence against the number of timeperiods for $P=2$ and $P=3$ respectively. We see that the results of numerical experiments are in excellent agreement with the theoretical expectations. Convergence in the two norms is nearly identical, except in the case of $P=2$ when using centered fluxes. In this case, the numerically observed short-time rate of convergence for the functional norm is $P+1$ while that for the discrete norm is $P+2$.

An important empirical observation from this experiment is that the longtime super-convergence rate of $2 P+q$ is exhibited by the FR scheme only after multiple thousand time periods depending on the polynomial order. In regard to applications, such a rate is relevant only for problems that 


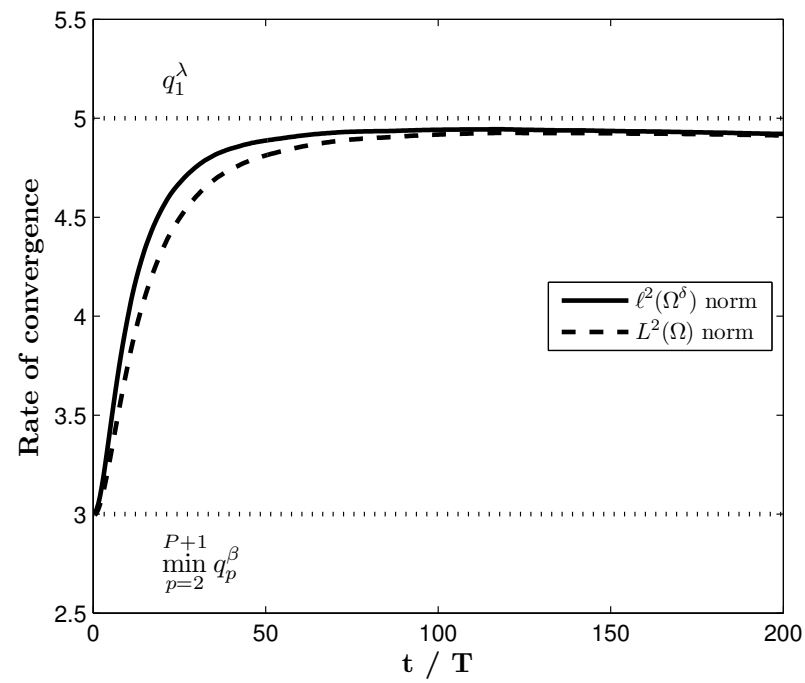

(a) Upwinded interface flux, $\alpha=0, \beta_{\mathrm{LDG}}=1 / 2, \tau_{\mathrm{LDG}}=0$.

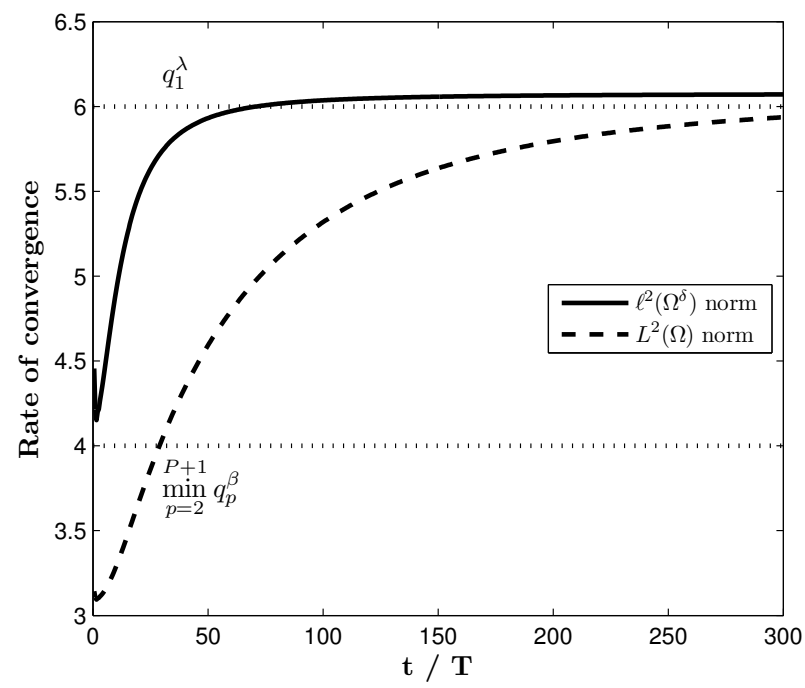

(b) Centered interface flux, $\alpha=1 / 2, \beta_{\mathrm{LDG}}=0, \tau_{\mathrm{LDG}}=0$.

Figure 7: 2-D advection-diffusion: Rate of convergence for the DG scheme of order $P=2$ on Gauss Legendre solution points. 


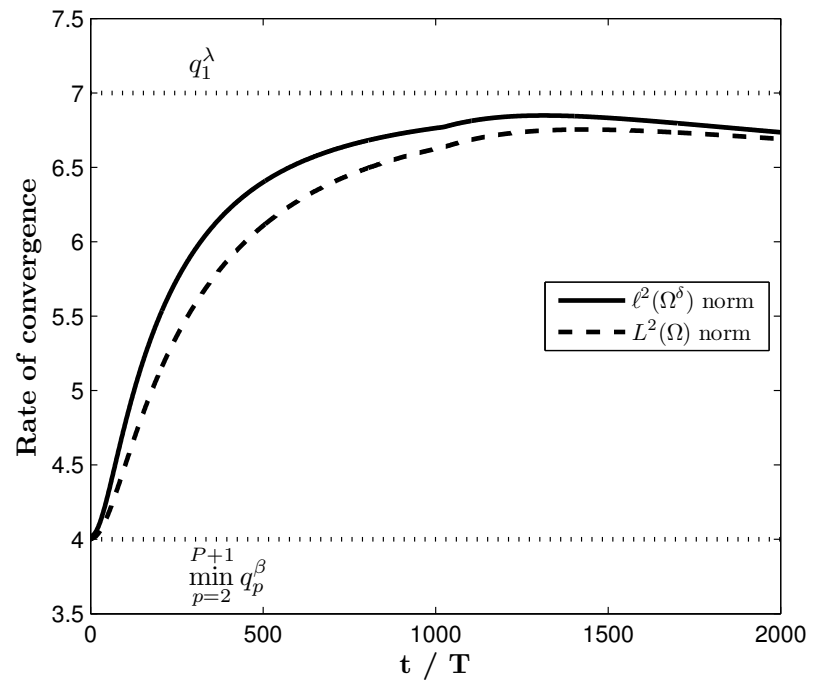

(a) Upwinded interface flux, $\alpha=0, \beta_{\mathrm{LDG}}=1 / 2, \tau_{\mathrm{LDG}}=0$.

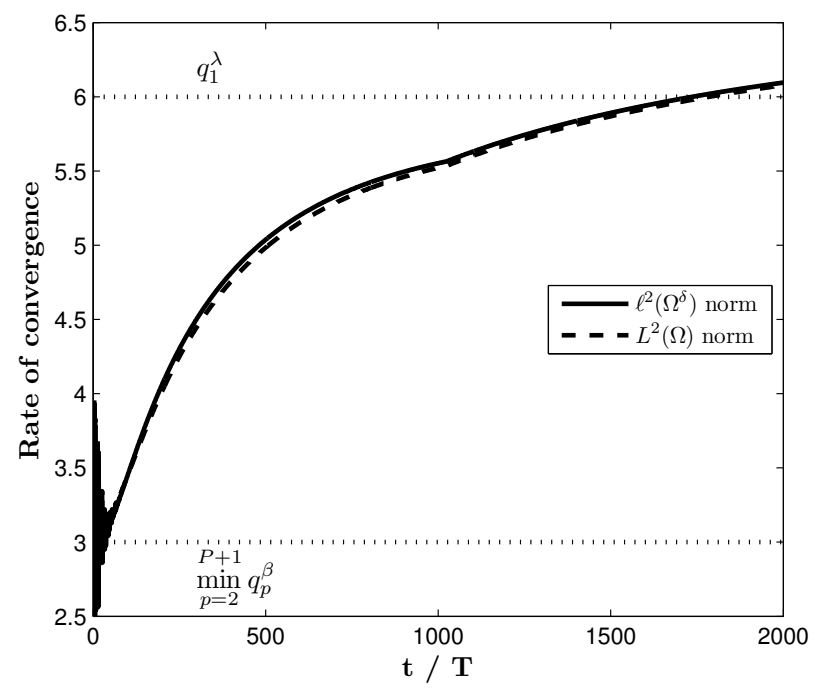

(b) Centered interface flux, $\alpha=1 / 2, \beta_{\mathrm{LDG}}=0, \tau_{\mathrm{LDG}}=0$.

Figure 8: 2-D advection-diffusion: Rate of convergence for the DG scheme of order $P=3$ on Gauss Legendre solution points. 
either have periodic boundary conditions or exhibit a quasi-steady solution of interest [42].

\subsection{Isentropic Euler vortex in $2 D$}

We now consider the Euler equations for a calorically perfect ideal gas,

$$
\frac{\partial}{\partial t}\left[\begin{array}{c}
\rho \\
\rho u \\
\rho v \\
\rho E
\end{array}\right]+\frac{\partial}{\partial x}\left[\begin{array}{c}
\rho u \\
\rho u^{2}+p \\
\rho u v \\
\rho H u
\end{array}\right]+\frac{\partial}{\partial y}\left[\begin{array}{c}
\rho v \\
\rho u v \\
\rho v^{2}+p \\
\rho H v
\end{array}\right]=\mathbf{0}, \quad \text { in } \Omega \subset \mathbb{R}^{2}, t>0,
$$

$$
p=(\gamma-1)\left(\rho E-\frac{1}{2} \rho\left(u^{2}+v^{2}\right)\right),
$$

where $\rho$ denotes density, $u, v$ denote the velocity components along $x$ and $y$ respectively, $E$ denotes total internal energy, $p$ denotes pressure, $H=E+p / \rho$ denotes total enthalpy, and $\gamma$ denotes the ratio of specific heats. For this experiment, we utilize the isentropic vortex test case introduced by Shu [44]. Herein, the initial condition consists of a uniform flow in the $y$ direction superposed with an isentropic vortex centered at the origin. Entropy is constant while the vorticity field decays exponentially in the radial direction. Following the prescription in [36],

$$
\begin{gathered}
{\left[\begin{array}{l}
\rho \\
u \\
v \\
p
\end{array}\right](x, y, 0)=\left[\begin{array}{c}
{\left[1-\frac{\gamma-1}{2}(\omega M R f)^{2}\right]^{\frac{1}{\gamma-1}}} \\
-\omega f y \\
1+\omega f x \\
\frac{1}{\gamma M^{2}} \rho(x, y, 0)^{\gamma}
\end{array}\right],} \\
\omega=\frac{\Gamma}{2 \pi R} \\
f=\exp \left(\frac{1-\left(x^{2}+y^{2}\right)}{2 R^{2}}\right)
\end{gathered}
$$

where $\Gamma$ is the strength of the vortex, $M$ is the free-stream Mach number and $R$ is a measure of the 'radius' of the vortex. For this experiment, we have used the values in [36] so that $\Gamma=13.5, M=0.4, R=1.5$.

The exact solution to this problem for an infinite domain, $\Omega=\mathbb{R}^{2}$, is the propagation of the vortex with unit velocity in the $y$ direction. In the numerical simulations, the domain is restricted to a square box, $[-40,40]^{2}$ with 
periodic boundary conditions imposed on the boundaries. Such periodicity effectively models a lattice of countably infinite vortex systems. While the interaction among vortices is weak, as the numerical solution advances in time, dispersion errors introduce spurious numerical wavefronts that propagate radially outwards, eventually leading to catastrophic non-linear interactions that cause a surge in the rate of error accumulation.

This problem is solved using DG, recovered via FR, for $P=2,3$ using Gauss-Legendre points as solution points. The numerical solution is marched in time using RK4 and the time-step is chosen small enough to ensure that errors due to numerical integration are much smaller than those due to spatial-discretization. Specifically, the time step is fixed at $2 \times 10^{-3}$ for $P=2$ and $10^{-3}$ for $P=3$. The common interface value is calculated using the fully-upwinded Lax-Friedrichs or Rusanov formula with $\alpha=0$. The rate of convergence is calculated at each time-step using two highly refined uniform grids with $260^{2}$ and $300^{2}$ elements for $P=2$, and $220^{2}$ and $260^{2}$ elements for $P=3$ respectively.

Figures 9 and 10 plot the evolution of error, in both norms and on both grids, for $P=2$ and $P=3$ respectively. The plots highlight three phases of error growth. The first phase, visible in Fig. 10, occurs during start-up, when $t / T$ is smaller than 1 , where $T=80$ is the time-period for the box. This phase is associated with the rapid decay of spurious modes during the initial few iterations leading to a loss of energy and corresponding error accumulation. The second phase, visible in both figures, is associated with the linear growth due to dispersion and dissipation in the physical mode. Both these phases are as expected from the dynamical system analysis in $\S 2$. However, for this non-linear problem, a third phase exists as well. Visible in Fig. 9, this phase occurs when $t / T>14$. It is associated with the non-linear interaction of spurious wavefronts generated by dispersion errors in each box of the lattice. Such catastrophic growth of error can be delayed either by refining the grid or by increasing the size of the numerical domain.

The distinction between the second and third phases can be observed further in Figs. 11 and 12 which plot contours of density for $P=2$ on the grid with $260^{2}$ elements at $t=10 T$ and $t=18 T$, respectively. Spurious numerical wavefronts, driven by dispersion errors, can be observed to propagate radially outward in Fig. 11b where the contours have been limited to a thin band about the background flow. These wavefronts interact due to the imposed periodicity, leading to spurious vortical structures that can be seen clearly in Fig. $12 \mathrm{~b}$ as well as 12a. 
Evolution of the rate of convergence for the two polynomial orders is plotted in Fig. 13. We have confined the time horizon to the first two phases of evolution where the behavior is essentially linear. Once again, the numerical results are in good agreement with the theoretical predictions. In particular, we see from Figs. 9 and 10 that the error for $P=3$ is several orders of magnitude lower than that for $P=2$. Consequently, the behavior is essentially linear and the evolution of the rate of convergence is similar to that for linear experiments performed in the previous subsection. This empirical observation further impresses the importance of using high-order methods for modern problems in fluid dynamics.

\section{Conclusions}

We have utilized Fourier analysis to rigorously establish that discontinuous finite element schemes formulated via Flux Reconstruction are consistent in regard to the linear advection problem, and if stable, converge to the exact solution in the limit of asymptotic resolution. The numerical solution for a scheme of polynomial order $P$ consists of a linear combination of $P+1$ eigenmodes. For a given mode, the eigenvalue governs numerical dispersion and dissipation, while the eigenvector governs the fraction of energy received by the mode from the initial condition. Of the $P+1$ modes, one and exactly one is 'physical' and the modal weights for the remaining $P$ 'spurious' modes vanish in the limit $k h \rightarrow 0$.

Such an eigensystem completely specifies the evolution of error, the vector norm of which is bounded above by a linear function of time. In this function, the constant term converges with grid refinement at a rate different from that of the linear term. Consequently, the rate of convergence for FR schemes is a function of time, starting from a short-time rate associated with polynomial interpolation, and asymptotically approaching a long-time rate associated with numerical differentiation. Both these rates can be inferred directly from the eigensystem of the numerical derivative operator.

The scope of future work is broad. A similar analysis of consistency and convergence for the diffusion equation can potentially provide further insight into the necessity of multiple corrections [43]. Similarly, generalization to the case of non-linear fluxes and non-tensor product elements would be of great value in evaluating the direct applicability of such analysis to real world problems. 


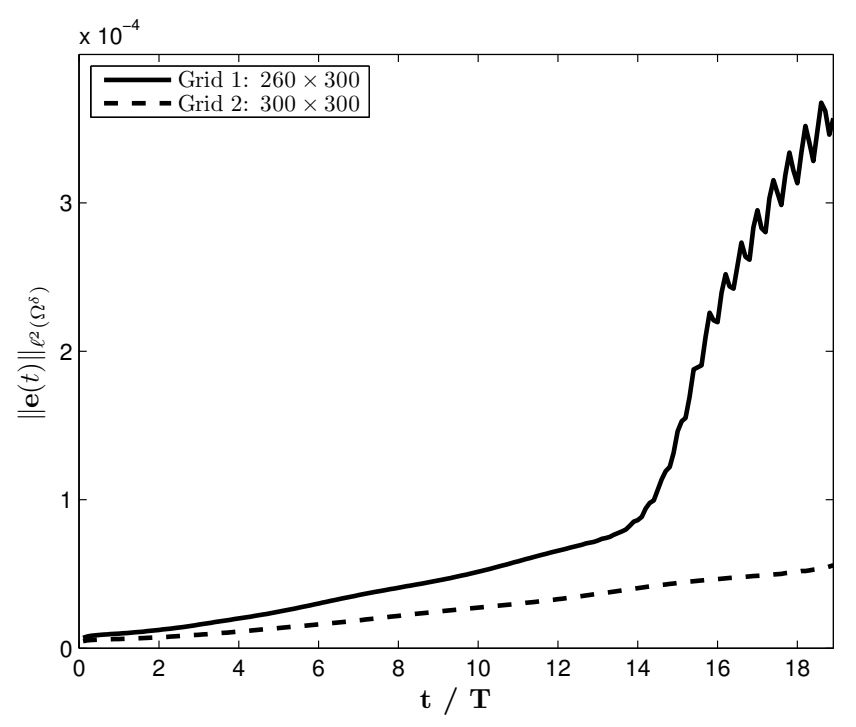

(a) $\ell^{2}\left(\Omega^{\delta}\right)$ norm

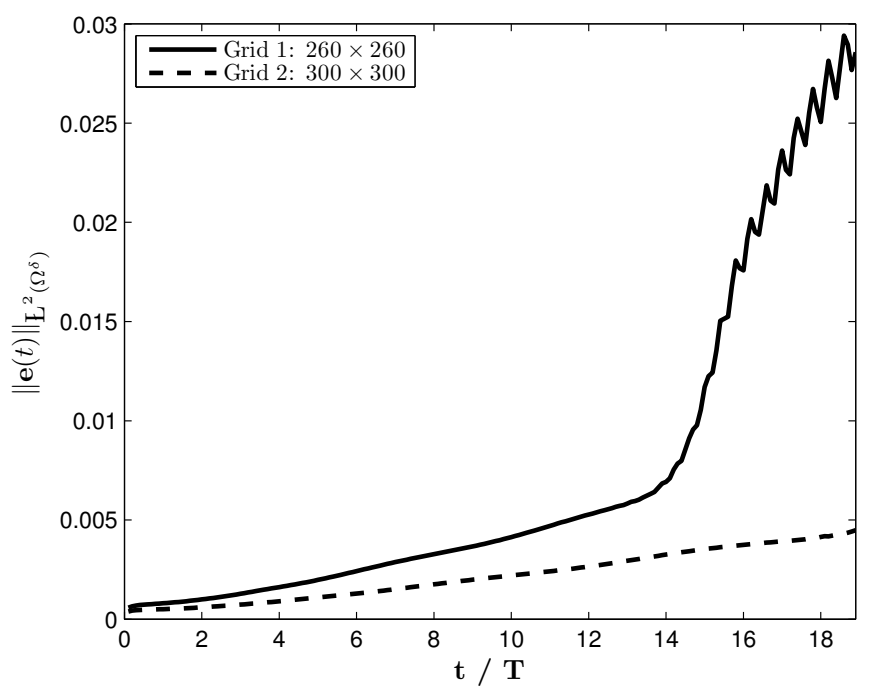

(b) $L^{2}(\Omega)$ norm

Figure 9: Isentropic vortex: Evolution of error for the DG scheme of polynomial order $P=2$ with upwinded flux, $\alpha=0$, on Gauss Legendre solution points. 


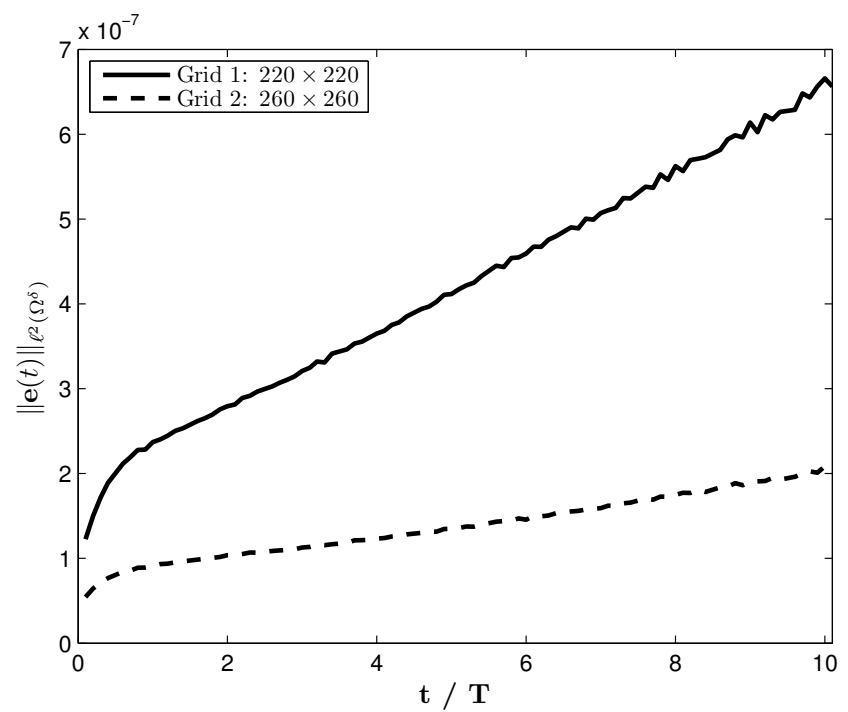

(a) $\ell^{2}\left(\Omega^{\delta}\right)$ norm

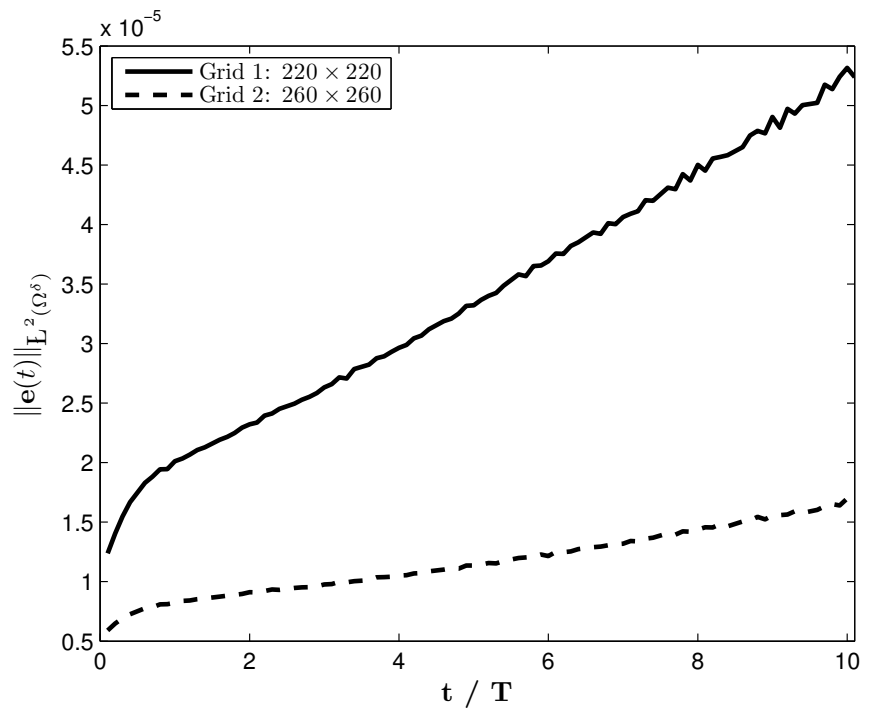

(b) $L^{2}(\Omega)$ norm

Figure 10: Isentropic vortex: Evolution of error for the DG scheme of polynomial order $P=3$ with upwinded flux, $\alpha=0$, on Gauss Legendre solution points. 


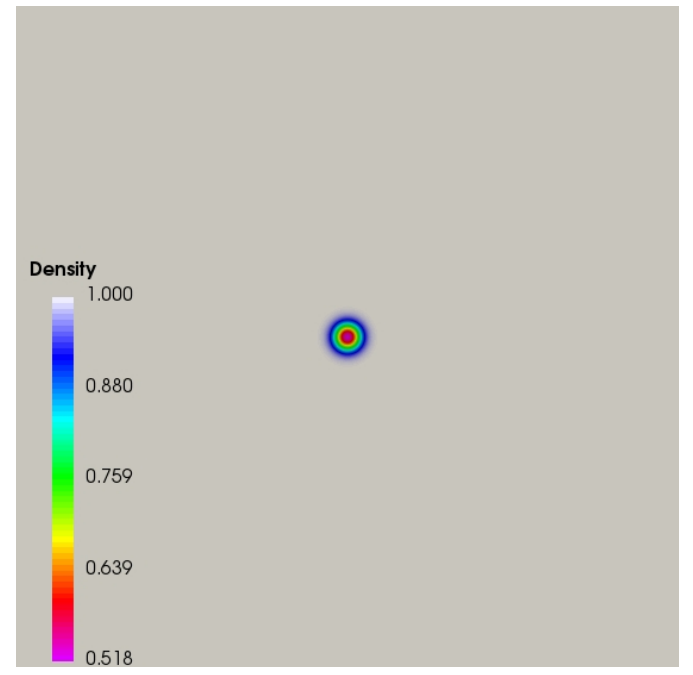

(a) Contours spanning the full solution

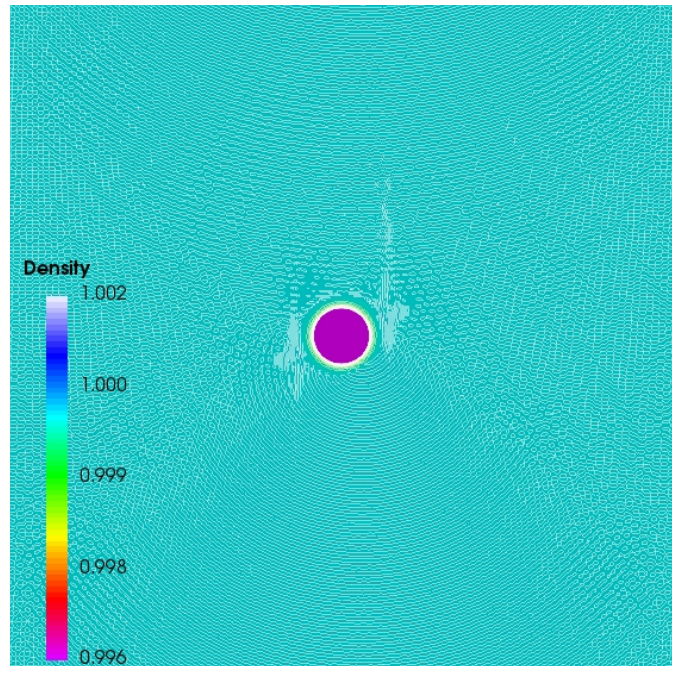

(b) Contours about background flow

Figure 11: Isentropic vortex: Contours of density for the upwinded DG scheme of polynomial order $P=2$ on Gauss Legendre solution points at $t=10 T$.

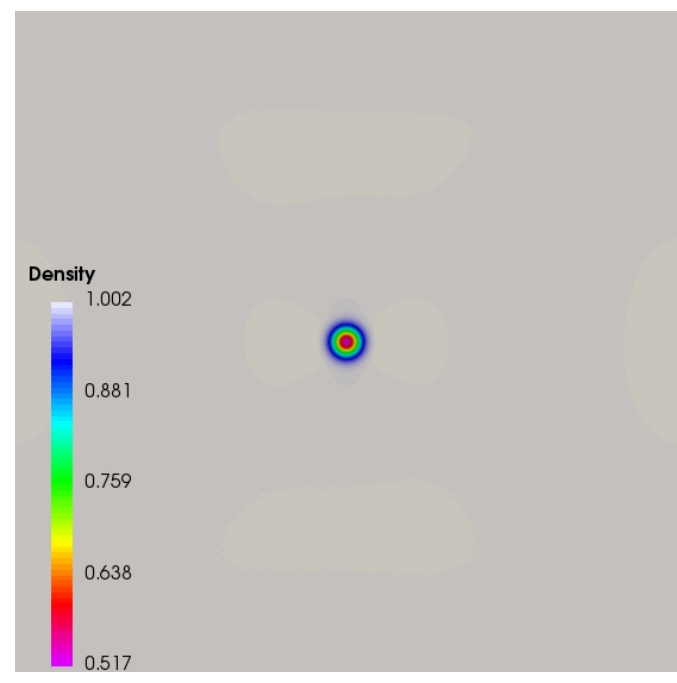

(a) Contours spanning the full solution

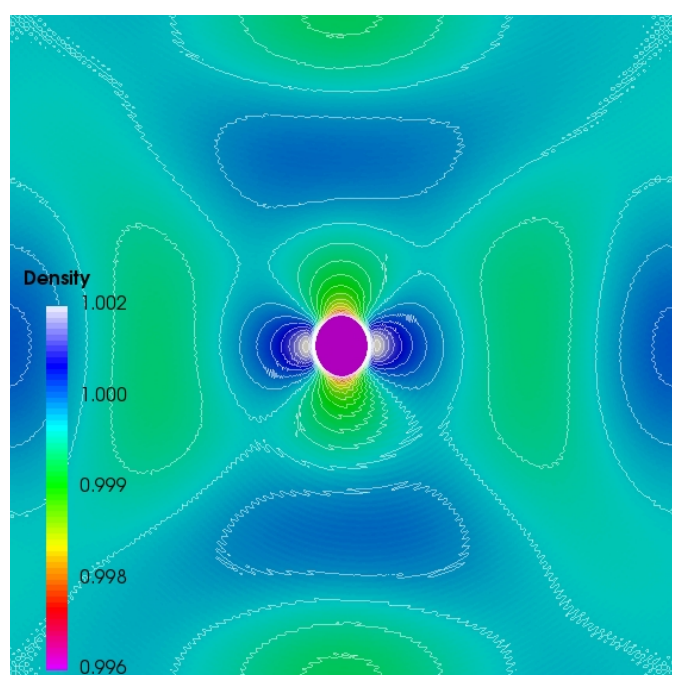

(b) Contours about background flow

Figure 12: Isentropic vortex: Contours of density for the upwinded DG scheme of polynomial order $P=2$ on Gauss Legendre solution points at $t=18 T$. 


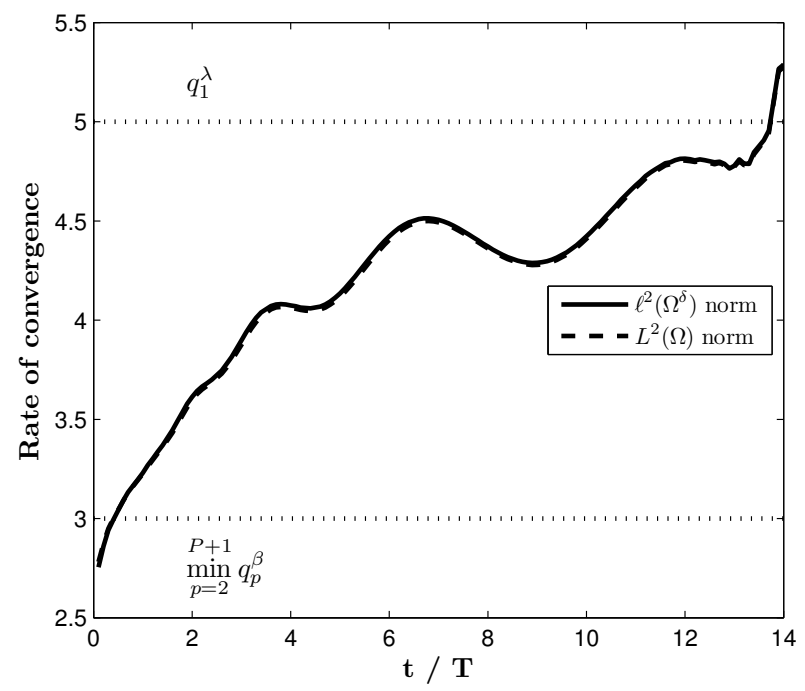

(a) $P=2$

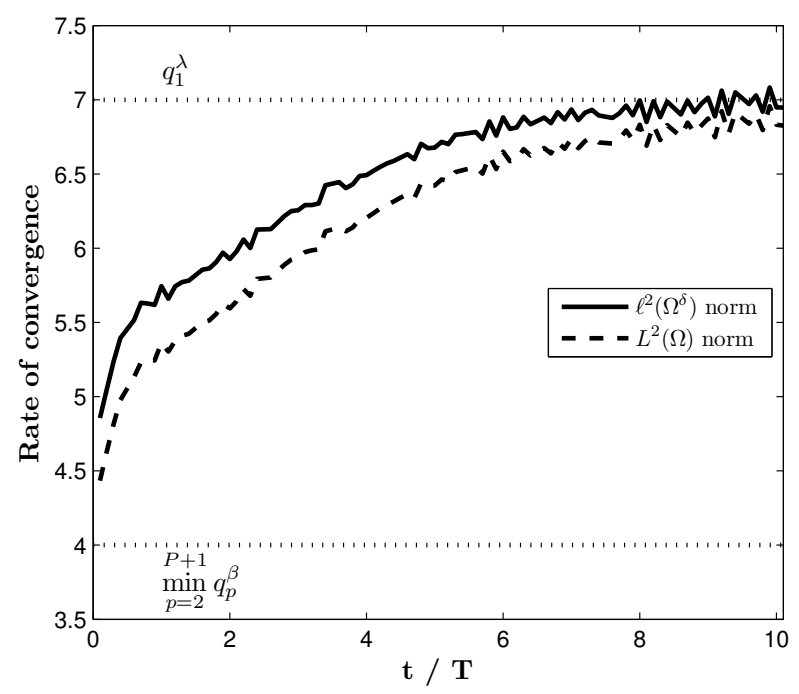

(b) $P=3$

Figure 13: Isentropic vortex: Rate of convergence for the DG scheme with upwinded flux, $\alpha=0$, on Gauss Legendre solution points. 


\section{Acknowledgments}

518 The authors acknowledge support from the Thomas V Jones Stanford ${ }_{519}$ Graduate Fellowship, the National Science Foundation Graduate Research ${ }_{520}$ Fellowship Program, and the Air Force Office of Scientific Research for their ${ }_{521}$ support via grant FA9550-14-1-0186. We are grateful to David Williams ${ }^{4}$ ${ }_{522}$ for his detailed comments regarding the manuscript.

\footnotetext{
${ }^{4}$ CFD Engineer, Computational Aerodynamic Optimization, Flight \& Vehicle Technology, Boeing Research \& Technology
} 
[1] Z. Haras, S. Ta'asan, Finite difference schemes for long-time integration, Journal of Computational Physics 114 (2) (1994) 265-279.

[2] S. K. Lele, Compact finite difference schemes with spectral-like resolution, Journal of Computational Physics 103 (1) (1992) 16-42.

[3] M. R. Visbal, D. V. Gaitonde, On the use of higher-order finite-difference schemes on curvilinear and deforming meshes, Journal of Computational Physics 181 (1) (2002) 155-185.

[4] J. S. Hesthaven, T. Warburton, Nodal discontinuous Galerkin methods: algorithms, analysis, and applications, Vol. 54, Springer Science \& Business Media, 2007.

[5] T. J. Hughes, The finite element method: linear static and dynamic finite element analysis, Courier Corporation, 2012.

[6] B. Cockburn, G. E. Karniadakis, C.-W. Shu, The development of discontinuous Galerkin methods, Springer, 2000.

[7] P. Lesaint, P.-A. Raviart, On a finite element method for solving the neutron transport equation, Mathematical aspects of finite elements in partial differential equations (33) (1974) 89-123.

[8] W. H. Reed, T. Hill, Triangular mesh methods for the neutron transport equation, Los Alamos Report LA-UR-73-479.

[9] D. A. Kopriva, J. H. Kolias, A conservative staggered-grid Chebyshev multidomain method for compressible flow, Tech. rep., DTIC Document (1995).

[10] Y. Liu, M. Vinokur, Z. Wang, Spectral difference method for unstructured grids I: basic formulation, Journal of Computational Physics 216 (2) (2006) 780-801.

[11] H. Huynh, A flux reconstruction approach to high-order schemes including discontinuous Galerkin methods, AIAA paper 4079 (2007) 2007.

[12] P. Castonguay, P. E. Vincent, A. Jameson, A new class of high-order energy stable flux reconstruction schemes for triangular elements, Journal of Scientific Computing 51 (1) (2012) 224-256. 
[13] D. Williams, P. Castonguay, P. E. Vincent, A. Jameson, Energy stable flux reconstruction schemes for advection-diffusion problems on triangles, Journal of Computational Physics 250 (2013) 53-76.

[14] D. Williams, A. Jameson, Energy stable flux reconstruction schemes for advection-diffusion problems on tetrahedra, Journal of Scientific Computing 59 (3) (2014) 721-759.

[15] H. Gao, Z. Wang, A conservative correction procedure via reconstruction formulation with the chain-rule divergence evaluation, Journal of Computational Physics 232 (1) (2013) 7-13.

[16] Z. Wang, H. Gao, A unifying lifting collocation penalty formulation including the discontinuous Galerkin, spectral volume/difference methods for conservation laws on mixed grids, Journal of Computational Physics 228 (21) (2009) 8161-8186.

[17] R. S. Falk, Analysis of finite element methods for linear hyperbolic problems, in: Discontinuous Galerkin Methods, Springer, 2000, pp. 103-112.

[18] C. Johnson, J. Pitkäranta, An analysis of the discontinuous galerkin method for a scalar hyperbolic equation, Mathematics of computation 46 (173) (1986) 1-26.

[19] T. Strouboulis, J. Oden, A posteriori error estimation of finite element approximations in fluid mechanics, Computer methods in applied mechanics and engineering 78 (2) (1990) 201-242.

[20] E. Süli, A posteriori error analysis and adaptivity for finite element approximations of hyperbolic problems, in: An introduction to recent developments in theory and numerics for conservation laws, Springer, 1999, pp. 123-194.

[21] S. Adjerid, K. D. Devine, J. E. Flaherty, L. Krivodonova, A posteriori error estimation for discontinuous galerkin solutions of hyperbolic problems, Computer methods in applied mechanics and engineering 191 (11) (2002) 1097-1112.

[22] B. Cockburn, M. Luskin, C.-W. Shu, E. Süli, Post-processing of Galerkin methods for hyperbolic problems, Springer, 2000. 
[23] P. E. Vincent, P. Castonguay, A. Jameson, A new class of high-order energy stable flux reconstruction schemes, Journal of Scientific Computing 47 (1) (2011) 50-72.

[24] A. Jameson, P. E. Vincent, P. Castonguay, On the non-linear stability of flux reconstruction schemes, Journal of Scientific Computing 50 (2) (2012) 434-445.

[25] P. Castonguay, D. Williams, P. Vincent, A. Jameson, Energy stable flux reconstruction schemes for advection-diffusion problems, Computer Methods in Applied Mechanics and Engineering 267 (2013) 400-417.

[26] C. K. Tam, J. C. Webb, Dispersion-relation-preserving finite difference schemes for computational acoustics, Journal of Computational Physics 107 (2) (1993) 262-281.

[27] L. L. Thompson, P. M. Pinsky, Complex wavenumber fourier analysis of the p-version finite element method, Computational Mechanics 13 (4) (1994) 255-275.

[28] M. Ainsworth, Discrete dispersion relation for hp-version finite element approximation at high wave number, SIAM Journal on Numerical Analysis 42 (2) (2004) 553-575.

[29] F. Q. Hu, H. L. Atkins, Eigensolution analysis of the discontinuous Galerkin method with nonuniform grids: I. one space dimension, Journal of Computational Physics 182 (2) (2002) 516-545.

[30] F. Q. Hu, H. L. Atkins, Two-dimensional wave analysis of the discontinuous Galerkin method with non-uniform grids and boundary conditions, in: Proceedings of the 8th AIAA/CEAS Aeroacoustics Conference, 2002.

[31] F. Q. Hu, M. Hussaini, P. Rasetarinera, An analysis of the discontinuous Galerkin method for wave propagation problems, Journal of Computational Physics 151 (2) (1999) 921-946.

[32] S. Sherwin, Dispersion analysis of the continuous and discontinuous Galerkin formulations, in: Discontinuous Galerkin Methods, Springer, 2000, pp. 425-431. 
[33] R. Moura, S. Sherwin, J. Peiro, Linear dispersion-diffusion analysis and its application to under-resolved turbulence simulations using discontinuous galerkin spectral/hp methods, Journal of Computational Physics 298 (2015) 695-710. doi:10.1016/j.jcp.2015.06.020. URL http://dx.doi.org/10.1016/j.jcp.2015.06.020

[34] M. Ainsworth, Dispersive and dissipative behaviour of high order discontinuous Galerkin finite element methods, Journal of Computational Physics 198 (1) (2004) 106-130.

[35] M. Ainsworth, P. Monk, W. Muniz, Dispersive and dissipative properties of discontinuous Galerkin finite element methods for the second-order wave equation, Journal of Scientific Computing 27 (1-3) (2006) 5-40.

[36] P. E. Vincent, P. Castonguay, A. Jameson, Insights from von neumann analysis of high-order flux reconstruction schemes, Journal of Computational Physics 230 (22) (2011) 8134-8154.

[37] K. Asthana, A. Jameson, High-order flux reconstruction schemes with minimal dispersion and dissipation, Journal of Scientific Computing (2014) 1-32.

[38] Y. Cheng, C.-W. Shu, Superconvergence and time evolution of discontinuous galerkin finite element solutions, Journal of Computational Physics 227 (22) (2008) 9612-9627.

[39] Y. Cheng, C.-W. Shu, Superconvergence of discontinuous galerkin and local discontinuous galerkin schemes for linear hyperbolic and convection-diffusion equations in one space dimension, SIAM Journal on Numerical Analysis 47 (6) (2010) 4044-4072.

[40] W. Guo, X. Zhong, J.-M. Qiu, Superconvergence of discontinuous galerkin and local discontinuous galerkin methods: Eigen-structure analysis based on fourier approach, Journal of Computational Physics 235 (2013) 458-485.

[41] L. Trefethen, D. Bau, Numerical Linear Algebra, Society for Industrial and Applied Mathematics, 1997.

URL https: //books . google. com/books?id=5Y1TPgAACAA J 
[42] H. L. Atkins, B. Helenbrook, Super-convergence of discontinuous galerkin method applied to the navier-stokes equations, AIAA Paper 3787 (2009) 2009.

[43] H. T. Huynh, A reconstruction approach to high-order schemes including discontinuous Galerkin for diffusion, AIAA paper 403 (2009) 2009.

[44] B. Cockburn, C. Johnson, C.-W. Shu, E. Tadmor, A. Quarteroni, Advanced numerical approximation of nonlinear hyperbolic equations: lectures given at the 2 nd session of the centro internazionale matematico estivo.

[45] E. Süli, D. Mayers, An Introduction to Numerical Analysis, Cambridge University Press, 2003.

URL https://books.google.com/books?id=hj9weaqJTbQC 


\section{Appendix A. Proofs of results in $\S 3$ and $\S 4$}

Proof of Lemma 3.1

Consider expanding $\boldsymbol{v}$ in the discrete monomial basis $\left\{\mathbf{1}, \boldsymbol{\xi}, \boldsymbol{\xi}^{\mathbf{2}}, \ldots, \boldsymbol{\xi}^{\boldsymbol{P}}\right\}$, where $\boldsymbol{\xi}^{\boldsymbol{i}}=\left(\xi_{p}\right)_{p=1,2, \ldots, P+1}^{i}$, as $\boldsymbol{v}=\sum_{i=0}^{P} a_{i} \boldsymbol{\xi}^{\boldsymbol{i}}$. Then,

$$
\begin{aligned}
\boldsymbol{D} \boldsymbol{v} & =\sum_{i=0}^{P} a_{i} \boldsymbol{D} \boldsymbol{\xi}^{\boldsymbol{i}} \\
& =\sum_{i=0}^{P} a_{i}\left(\sum_{m=0}^{P} \frac{\mathrm{d} \ell_{m}}{\mathrm{~d} \xi}\left(\xi_{p}\right) \xi_{m}^{i}\right)_{p=1,2, \ldots, P+1} \\
& =\sum_{i=0}^{P} a_{i}\left(\frac{\mathrm{d}}{\mathrm{d} \xi}\left(\sum_{m=0}^{P} \ell_{m}(\xi) \xi_{m}^{i}\right)\left(\xi_{p}\right)\right)_{p=1,2, \ldots, P+1} \\
& =\sum_{i=0}^{P} a_{i}\left(\frac{\mathrm{d}}{\mathrm{d} \xi}\left(\xi^{i}\right)\left(\xi_{p}\right)\right)_{p=1,2, \ldots, P+1} \\
& =\sum_{i=1}^{P} a_{i} i \boldsymbol{\xi}^{\boldsymbol{i}-\mathbf{1}},
\end{aligned}
$$

which is the vector obtained from sampling $\frac{d v}{d \xi}$ at the $P+1$ points.

\section{Proof of Lemma 3.2}

Let $\boldsymbol{v} \in \mathbb{C}^{(P+1) \times 1}$ be an eigenvector of $\boldsymbol{D}$, so that $\boldsymbol{D} \boldsymbol{v}=\gamma \boldsymbol{v}$ and $\|\boldsymbol{v}\|_{2}=1$. Consider expanding $\boldsymbol{v}$ in the discrete monomial basis as $\boldsymbol{v}=\sum_{i=0}^{P} a_{i} \boldsymbol{\xi}^{i}$. Then, using Lemma 3.1, $\sum_{i=1}^{P} a_{i} i \boldsymbol{\xi}^{\boldsymbol{i - 1}}=\gamma \sum_{i=0}^{P} a_{i} \boldsymbol{\xi}^{\boldsymbol{i}}$, which can be rewritten as

$$
\sum_{i=0}^{P}\left(a_{i+1}(i+1)-\gamma a_{i}\right) \boldsymbol{\xi}^{i}=\mathbf{0}
$$

where $a_{P+1}:=0$. Let $\boldsymbol{b}$ be such that $b_{i}=a_{i+1}(i+1)-\gamma a_{i}$ for $i=0,1, \ldots, P$ and introduce the Vandermonde matrix $\boldsymbol{V}=\left[\mathbf{1}, \boldsymbol{\xi}, \ldots, \boldsymbol{\xi}^{\boldsymbol{P}}\right]$ so that Eqn. (A.2) can be recognized as $\boldsymbol{V} \boldsymbol{b}=\mathbf{0}$, which yields the unique trivial solution $\boldsymbol{b}=\mathbf{0}$ 
since the $P+1$ solution points are assumed to be distinct. This results in the backward recursive relation,

$$
\begin{cases}a_{i}=0 \quad \forall i & \gamma \neq 0 \\ a_{0}=\frac{1}{\sqrt{P+1}}, a_{i}=0 \text { for } i=1, \ldots, P & \gamma=0\end{cases}
$$

where the factor of $\frac{1}{\sqrt{P+1}}$ arises from the fact that the eigenvectors are unit vectors. Hence, there is a single non-zero eigenvector $\frac{1}{\sqrt{P+1}} \mathbf{1}$ and the corresponding eigenvalue is given by $\gamma=0$.

\section{Proof of Lemma 3.3}

This can be shown by noting that the spectral radius of any matrix is bounded by its norm,

$$
\begin{aligned}
& \max _{p=1}^{P+1}\left|\gamma_{p}\right|=\max _{p=1}^{P+1}\left\|\gamma_{p} \boldsymbol{w}_{p}\right\|_{2} \\
& =\max _{p=1}^{P+1}\left\|\boldsymbol{Q}(k h) \boldsymbol{w}_{p}\right\|_{2} \\
& \leq \max _{\boldsymbol{y},\|\boldsymbol{y}\|_{2}=1}\|\boldsymbol{Q}(k h) \boldsymbol{y}\|_{2} \\
& =\|\boldsymbol{Q}(k h)\|_{2} \\
& =\left\|(1-\alpha) \boldsymbol{g}_{\boldsymbol{L}, \boldsymbol{\xi}}\left(\exp (-i k h) \boldsymbol{\ell}_{+}^{T}-\boldsymbol{\ell}_{-}^{T}\right)+\boldsymbol{D}+\alpha \boldsymbol{g}_{\boldsymbol{R}, \boldsymbol{\xi}}\left(\exp (i k h) \boldsymbol{\ell}_{-}^{T}-\boldsymbol{\ell}_{+}^{T}\right)\right\|_{2} \\
& \leq\left((1-\alpha)\left\|\boldsymbol{g}_{\boldsymbol{L}, \boldsymbol{\xi}}\right\|_{2}+\alpha\left\|\boldsymbol{g}_{\boldsymbol{R}, \boldsymbol{\xi}}\right\|_{2}\right)\left(\left\|\boldsymbol{\ell}_{+}\right\|_{2}+\left\|\boldsymbol{\ell}_{-}\right\|_{2}\right)+\|\boldsymbol{D}\|_{F} \\
& \leq 2\left(\sqrt{\sum_{p=1}^{P+1} g_{L, \xi_{p}}^{2}}+\sqrt{\sum_{p=1}^{P+1} g_{R, \xi_{p}}^{2}}\right) \sqrt{\sum_{p=1}^{P+1} \ell_{p}^{2}(+1)}+\sqrt{\sum_{p=1}^{P+1} \sum_{q=1}^{P+1}\left|\frac{\mathrm{d} \ell_{q}}{\mathrm{~d} \xi}\left(\xi_{p}\right)\right|^{2}}
\end{aligned}
$$

where the subscript $F$ denotes the Frobenius norm. The boundedness of Lagrange polynomials and their derivatives follows from the assumptions that $P$ is finite, the solutions points are distinct and the parent domain is bounded.

Proof of Lemma 3.4

The proof follows from Eqn. (11) by observing that $\mathbf{1}$ belongs to the null space of $\boldsymbol{Q}(0)$,

$$
\boldsymbol{Q}(0) \mathbf{1}=\left[\left((1-\alpha) \boldsymbol{g}_{\boldsymbol{L}, \boldsymbol{\xi}}-\alpha \boldsymbol{g}_{\boldsymbol{R}, \boldsymbol{\xi}}\right)\left(\boldsymbol{\ell}_{+}^{T}-\boldsymbol{\ell}_{-}^{T}\right)+\boldsymbol{D}\right] \mathbf{1}=\mathbf{0},
$$


where we have used Lemma $3.2, \boldsymbol{D} \mathbf{1}=\mathbf{0}$, and extrapolation property of the Lagrange operators, $\ell_{-}^{T} \mathbf{1}=\ell_{+}^{T} \mathbf{1}=1$.

Proof of Lemma 3.5

Taking limits in Eqn. (15) and using Eqn. (6),

$$
\boldsymbol{W}(0) \boldsymbol{\beta}(0)=\boldsymbol{w}_{0}(0)=\mathbf{1}
$$

Since $\boldsymbol{W}(0)$ is invertible by the assumption of diagonalizability, therefore $\boldsymbol{\beta}(0)$ is unique. Also, from Lemma 3.4, we know that

$$
\boldsymbol{W}(0)\left(\sqrt{P+1} \boldsymbol{e}_{1}\right)=\sqrt{P+1} \boldsymbol{w}_{1}(0)=\mathbf{1},
$$

which shows that the one and only solution is given by $\beta_{p}(0)=\sqrt{P+1} \delta_{p 1}$ for $p=1,2, \ldots, P+1$. This also shows that there exists exactly one physical mode.

\section{Proof of Lemma 3.6}

Consider the numerical derivative vector obtained when the numerical differentiation operator acts on the initial condition, $\boldsymbol{Q}(k h) \exp \left(i k x_{n}\right) \boldsymbol{w}_{0}$. From Eqns. (14), (15),

$$
\begin{aligned}
\boldsymbol{Q}(k h) \boldsymbol{w}_{0} & =\boldsymbol{Q}(k h) \boldsymbol{W}(k h) \boldsymbol{\beta}(k h) \\
& =\boldsymbol{W}(k h) \boldsymbol{\Gamma}(k h) \boldsymbol{\beta}(k h) \\
& =\sum_{p=1}^{P+1} \beta_{p}(k h) \gamma_{p}(k h) \boldsymbol{w}_{p}(k h) \\
& =\beta_{1}(k h) \gamma_{1}(k h) \boldsymbol{w}_{1}(k h)+\sum_{p=2}^{P+1} \beta_{p}(k h) \gamma_{p}(k h) \boldsymbol{w}_{p}(k h),
\end{aligned}
$$

so that by Lemmas 3.3, 3.4 and 3.5,

$$
\boldsymbol{Q}(k h) \boldsymbol{w}_{0} \rightarrow \gamma_{1}(k h) \mathbf{1}=i k \frac{h}{2} \lambda_{1}(0) \mathbf{1} \text { as } k h \rightarrow 0
$$


Alternatively, consider expanding $\boldsymbol{Q}(k h) \boldsymbol{w}_{0}$ via the Taylor series, using Eqns. (6) and (11), and retaining only the first order terms,

$$
\begin{aligned}
\boldsymbol{Q}(k h) \boldsymbol{w}_{0} & =\left(\boldsymbol{Q}(0)-i k h\left((1-\alpha) \boldsymbol{g}_{\boldsymbol{L}, \boldsymbol{\xi}} \boldsymbol{\ell}_{+}^{T}-\alpha \boldsymbol{g}_{\boldsymbol{R}, \boldsymbol{\xi}} \boldsymbol{\ell}_{-}^{T}\right)\right)\left(\mathbf{1}+i k \frac{h}{2}(\mathbf{1}+\boldsymbol{\xi})\right)+\mathcal{O}(k h)^{2} \\
& =\left(1+i k \frac{h}{2}\right) \boldsymbol{Q}(0) \mathbf{1}+i k \frac{h}{2}\left[\boldsymbol{Q}(0) \boldsymbol{\xi}-2\left((1-\alpha) \boldsymbol{g}_{\boldsymbol{L}, \boldsymbol{\xi}} \boldsymbol{\ell}_{+}^{T}-\alpha \boldsymbol{g}_{\boldsymbol{R}, \boldsymbol{\xi}} \boldsymbol{\ell}_{-}^{T}\right) \mathbf{1}\right]+\mathcal{O}(k h)^{2} \\
& =i k \frac{h}{2}\left[\boldsymbol{Q}(0) \boldsymbol{\xi}-2\left((1-\alpha) \boldsymbol{g}_{\boldsymbol{L}, \boldsymbol{\xi}}-\alpha \boldsymbol{g}_{\boldsymbol{R}, \boldsymbol{\xi}}\right)\right]+\mathcal{O}(k h)^{2},
\end{aligned}
$$

where we have used Lemma 3.4. Also, from the properties of $\boldsymbol{\ell}_{-}, \boldsymbol{\ell}_{+}$and $\boldsymbol{D}$, we have, $\boldsymbol{Q}(0) \boldsymbol{\xi}=\left(2\left((1-\alpha) \boldsymbol{g}_{\boldsymbol{L}, \boldsymbol{\xi}}-\alpha \boldsymbol{g}_{\boldsymbol{R}, \boldsymbol{\xi}}\right)+\mathbf{1}\right) \mathbb{I}_{P>0}$, so that,

$$
\boldsymbol{Q}(k h) \boldsymbol{w}_{0}= \begin{cases}i k \frac{h}{2} \mathbf{1}+\mathcal{O}(k h)^{2} & P>0 \\ -i k h\left((1-\alpha) g_{L, \xi}-\alpha g_{R, \xi}\right)+\mathcal{O}(k h)^{2} & P=0\end{cases}
$$

Now note that the correction functions for $P=0$ are completely specified by the boundary constraints, Eqn. $(9)$, so that $g_{L}(\xi)=\frac{1}{2}(1-\xi), g_{R, \xi}=\frac{1}{2}(1+\xi)$. Hence,

$$
\boldsymbol{Q}(k h) \boldsymbol{w}_{0} \rightarrow i k \frac{h}{2} \mathbf{1} \text { as } k h \rightarrow 0, \forall P .
$$

Comparing Eqn. (A.12) with Eqn. (A.9), we get $\lambda_{1} \rightarrow 1$ as $k h \rightarrow 0$.

\section{Proof of Theorem 3.1}

For a consistent FR scheme, the numerical conservation law, Eqn. (3), must converge to the vectorized form of the exact conservation law, Eqn. (1), in the asymptotic limit. Since integration in time is assumed to be perfect, we require that the numerical derivative vector in the parent space, $\boldsymbol{Q}(k h) \boldsymbol{u}_{n}^{\boldsymbol{\delta}}(t)$, converges to the exact derivative values at the solution points,

$$
\begin{aligned}
\frac{\partial u_{n}}{\partial \xi}\left(\boldsymbol{x}_{n}, t\right) & =\exp \left(i k x_{n}\right) \exp (-i k c t) i k \frac{h}{2} \boldsymbol{w}_{0} \\
& \rightarrow \exp \left(i k x_{n}\right) \exp (-i k c t) i k \frac{h}{2} \mathbf{1} \text { as } k h \rightarrow 0
\end{aligned}
$$


In order to prove this, we can use Eqns. (14) and (16), so that

$$
\begin{aligned}
\boldsymbol{Q}(k h) \boldsymbol{u}_{n}^{\boldsymbol{\delta}}(t) & =\boldsymbol{Q}(k h) \exp \left(i k x_{n}\right) \sum_{p=1}^{P+1} \exp \left(-i k c \lambda_{p} t\right) \beta_{p} \boldsymbol{w}_{p} \\
& =\exp \left(i k x_{n}\right) \sum_{p=1}^{P+1} \exp \left(-i k c \lambda_{p} t\right) \beta_{p} \gamma_{p} \boldsymbol{w}_{p}
\end{aligned}
$$

Next, we pass to the asymptotic limit $k h \rightarrow 0$, and utilize the results from Lemmas 3.3, 3.4 and 3.5, so that

$$
\boldsymbol{Q}(k h) \boldsymbol{u}_{n}^{\boldsymbol{\delta}}(t) \rightarrow \exp \left(i k x_{n}\right) \exp \left(-i k c \lambda_{1} t\right) \gamma_{1} \mathbf{1} \text { as } k h \rightarrow 0 .
$$

Finally, Lemma 3.6 provides us with the limit on $\lambda_{1}$, so that

$$
\boldsymbol{Q}(k h) \boldsymbol{u}_{n}^{\boldsymbol{\delta}}(t) \rightarrow \exp \left(i k x_{n}\right) \exp (-i k c t) i k \frac{h}{2} \mathbf{1} \text { as } k h \rightarrow 0 .
$$

695

Proof of Theorem 3.2

Using Eqn. (19),

$$
\begin{aligned}
\left\|\boldsymbol{e}_{n}(t)\right\|_{2}= & \left\|\sum_{p=1}^{P+1}\left[\exp \left(k c \lambda_{p}^{\operatorname{Im}} t\right) \exp \left(-i k c\left(\lambda_{p}^{\mathrm{Re}}-1\right) t\right)-1\right] \beta_{p} \boldsymbol{w}_{p}\right\|_{2} \\
\leq & \sum_{p=1}^{P+1}\left|\exp \left(k c \lambda_{p}^{\mathrm{Im}} t\right) \exp \left(-i k c\left(\lambda_{p}^{\mathrm{Re}}-1\right) t\right)-1\right|\left|\beta_{p}\right| \\
\leq & \left|\exp \left(k c \lambda_{1}^{\mathrm{Im}} t\right) \exp \left(-i k c\left(\lambda_{1}^{\mathrm{Re}}-1\right) t\right)-1\right|\left|\beta_{1}\right| \\
& +\sum_{p=2}^{P+1}\left(\left|\exp \left(k c \lambda_{p}^{\operatorname{Im}} t\right) \exp \left(-i k c\left(\lambda_{p}^{\mathrm{Re}}-1\right) t\right)\right|+1\right)\left|\beta_{p}\right| .
\end{aligned}
$$

Now observe that $\left|\exp \left(-i k c\left(\lambda_{p}^{\mathrm{Re}}-1\right) t\right)\right|=1$ and $\left|\exp \left(k c \lambda_{p}^{\operatorname{Im}} t\right)\right| \leq 1$ by the stability criterion on eigenvalues, Eqn. (20), so that by the triangle inequality,

$$
\left\|\boldsymbol{e}_{n}(t)\right\|_{2} \leq\left|\exp \left(k c \lambda_{1}^{\operatorname{Im}} t\right) \exp \left(-i k c\left(\lambda_{1}^{\mathrm{Re}}-1\right) t\right)-1\right|\left|\beta_{1}\right|+2 \sum_{p=2}^{P+1}\left|\beta_{p}\right|,
$$


which can be simplified via the convergence of $\boldsymbol{\beta}$, Lemma 3.5, as

$$
\lim _{k h \rightarrow 0}\left\|\boldsymbol{e}_{n}(t)\right\|_{2} \leq \lim _{k h \rightarrow 0}\left|\exp \left(-i k c\left(\lambda_{1}^{\operatorname{Re}}(k h)-1\right) t\right)-1\right| \sqrt{P+1}
$$

Now, under the conditions of Lemma 3.6, $\lambda_{1} \rightarrow 1$, yielding $\lim _{k h \rightarrow 0}\left\|\boldsymbol{e}_{n}(t)\right\|_{2}=0$.

Proof of Lemma 3.7

Using the analytical form of the exact solution,

$$
\begin{aligned}
u(x, t) & =\mathcal{P}(u(x, t))+(1-\mathcal{P})(\exp (i k(x-c t))), \quad x \in \Omega_{n}, \\
& =\mathcal{P}(u(x, t))+\exp \left(i k\left(x_{n}-c t\right)\right)(1-\mathcal{P})\left(\exp \left(i k \frac{h}{2}(1+\xi)\right)\right), \quad x \in \Omega_{n},
\end{aligned}
$$

where $\mathcal{P}$ denotes the collocation projection operator that interpolates its argument at set of solution points in the $n^{\text {th }}$ element $\left(\xi_{p}\right)$ for $p=1,2, \ldots, P+$ 1 ,

$$
\mathcal{P}\left(u\left(x \in \Omega_{n}, t\right)\right)=\sum_{p=1}^{P+1} u_{n, p}(t) \ell_{n, p}(x)
$$

From standard error estimates in Lagrange interpolation [45],

$$
\begin{aligned}
\left|(1-\mathcal{P})\left(\exp \left(i k \frac{h}{2}(1+\xi)\right)\right)\right| & \leq \sup _{\phi \in[-1,1]}\left|\left(i k \frac{h}{2}\right)^{P+1} \exp \left(i k \frac{h}{2}(1+\phi)\right) \frac{\prod_{q=1}^{P+1}\left(\xi-\xi_{q}\right)}{(P+1) !}\right| \\
& \leq a(P, \boldsymbol{\xi})(k h)^{P+1} .
\end{aligned}
$$

for some constant $a$ that depends on $P$ and $\boldsymbol{\xi}$. Then, from the polynomial form of the numerical solution, Eqn. (2), we get

$$
\left|u^{\delta}\left(x \in \Omega_{n}, t\right)-u\left(x \in \Omega_{n}, t\right)\right| \leq\left|\sum_{p=1}^{P+1}\left(u_{n, p}^{\delta}(t)-u_{n, p}(t)\right) \ell_{n, p}(x)\right|+|a(P, \boldsymbol{\xi})|(k h)^{P+1} .
$$

The proof now follows from Theorem 3.2. 
We begin by rewriting Eqn. (A.18) using the series expansion for an exponential and the triangle inequality,

$$
\begin{aligned}
\left\|\boldsymbol{e}_{n}(t)\right\|_{2} & \leq\left|\exp \left(-i k c\left(\lambda_{1}(k h)-1\right) t\right)-1\right|\left|\beta_{1}(k h)\right|+2 \sum_{p=2}^{P+1}\left|\beta_{p}(k h)\right| \\
& =\left|\sum_{j=1}^{\infty} \frac{\left(-i k c\left(\lambda_{1}(k h)-1\right) t\right)^{j}}{j !}\right|\left|\beta_{1}(k h)\right|+2 \sum_{p=2}^{P+1}\left|\beta_{p}(k h)\right| \\
& \leq \sum_{j=1}^{\infty} \frac{\left(k c\left|\lambda_{1}(k h)-1\right| t\right)^{j}}{j !}\left|\beta_{1}(k h)\right|+2 \sum_{p=2}^{P+1}\left|\beta_{p}(k h)\right| \\
& =c k\left|\lambda_{1}(k h)-1\right| t\left(1+\sum_{j=1}^{\infty} \frac{\left(k c\left|\lambda_{1}(k h)-1\right| t\right)^{j}}{(j+1) !}\right)\left|\beta_{1}(k h)\right|+2 \sum_{p=2}^{P+1}\left|\beta_{p}(k h)\right| .
\end{aligned}
$$

Next, we pass to the asymptotic limit of $k h \rightarrow 0$ for $k>0$ and a fixed time $t$. Lemma 3.6 ensures that $\lambda_{1} \rightarrow 1$, so that

$$
\begin{aligned}
\left(1+\sum_{j=1}^{\infty} \frac{\left(k c\left|\lambda_{1}(k h)-1\right| t\right)^{j}}{(j+1) !}\right) & \leq 1+\left|\lambda_{1}(k h)-1\right| \sum_{j=1}^{\infty} \frac{(k c t)^{j}}{(j+1) !} \\
& \leq 1+\left|\lambda_{1}(k h)-1\right| \sum_{j=1}^{\infty} \frac{(k c t)^{j}}{j !} \\
& =1+\left|\lambda_{1}(k h)-1\right|(\exp (k c t)-1) \\
& \rightarrow 1 .
\end{aligned}
$$


Finally, substituting Eqns. (A.25), (28), and (29) in Eqn. (A.24), we get

$$
\begin{aligned}
\left\|\boldsymbol{e}_{n}(t)\right\|_{2} & \leq C_{1} c k(k h)^{q_{1}^{\lambda}} t\left(\sqrt{P+1}+C_{2}(k h)^{q_{1}^{\beta}}\right)+2 C_{2} \sum_{p=2}^{P+1}(k h)^{q_{p}^{\beta}} \\
& \leq C_{1} \sqrt{P+1} c k(k h)^{q_{1}^{\lambda}} t+2 C_{2} \sum_{p=2}^{P+1}(k h)^{q_{p}^{\beta}} \\
& =C_{1} \sqrt{P+1} c k(k h)^{q_{1}^{\lambda}} t+2 C_{2}(k h)^{\min _{p=2}^{P+1} q_{p}^{\beta}} \sum_{p=2}^{P+1}(k h)^{q_{p}^{\beta}-\min _{p=2}^{P+1} q_{p}^{\beta}} \\
& \leq C_{1} \sqrt{P+1} c k(k h)^{q_{1}^{\lambda}} t+2 C_{2}(k h)^{\min _{p=2}^{P+1} q_{p}^{\beta}}
\end{aligned}
$$

710 as $k h \rightarrow 0$. 